\title{
Using Synchronic and Diachronic Relations for Summarizing Multiple Documents Describing Evolving Events
}

\author{
Stergos D. Afantenos ${ }^{* \dagger} \quad$ Vangelis Karkaletsis ${ }^{\ddagger}$ \\ Panagiotis Stamatopoulos ${ }^{\S} \quad$ Constantin Halatsis ${ }^{\S}$
}

\begin{abstract}
In this paper we present a fresh look at the problem of summarizing evolving events from multiple sources. After a discussion concerning the nature of evolving events we introduce a distinction between linearly and non-linearly evolving events. We present then a general methodology for the automatic creation of summaries from evolving events. At its heart lie the notions of Synchronic and Diachronic cross-document Relations (SDRs), whose aim is the identification of similarities and differences between sources, from a synchronical and diachronical perspective. SDRs do not connect documents or textual elements found therein, but structures one might call messages. Applying this methodology will yield a set of messages and relations, SDRs, connecting them, that is a graph which we call grid. We will show how such a grid can be considered as the starting point of a Natural Language Generation System. The methodology is evaluated in two case-studies, one for linearly evolving events (descriptions of football matches) and another one for non-linearly evolving events (terrorist incidents involving hostages). In both cases we evaluate the results produced by our computational systems.
\end{abstract}

\section{Introduction}

Exchange of information is vital for the survival of human beings. It has taken many forms throughout the history of mankind ranging from gossiping (Pinker 1997) to the publication of news via highly sophisticated media. Internet provides us with new perspectives, making the exchange of information not only easier than ever, but also virtually unrestricted.

Yet, there is a price to be paid to this richness of means, as it is difficult to assimilate this plethora of information in a small amount of time. Suppose a person would like to keep track of the evolution of an event via its description available over the Internet. There is such a vast body of data (news) relating

\footnotetext{
${ }^{\dagger}$ Laboratoire d'Informatique Fondamentale de Marseille, Centre National de la Recherche Scientific (LIF - CNRS - UMR 6166)

‡Institute of Informatics and Telecommunications, NCSR "Demokritos", Athens, Greece.

$\S$ Department of Informatics and Telecommunications, National and Kapodistrian University of Athens, Athens, Greece.

${ }^{*}$ Corresponding author; email: stergos . af antenos@lif .univ-mrs.fr
} 
to the event that it is practically impossible to read all of them and decide which are really of interest. A simple visit at, let's say, Google News ${ }^{1}$ will show that for certain events the number of hits, i.e. related stories, amounts to the thousands. Hence it is simply impossible to scan through all these documents, compare them for similarities and differences, while reading through in order to follow the evolution of the event.

Yet, there might be an answer to this problem: automatically produced (parametrizable) text summaries. This is precisely the issue we will be concerned with in this paper. We will focus on Evolving Summarization; or, to be more precise, the automatic summarization of events evolving throughout time.

While there has been pioneering work on automatic text summarization more than 30 years ago, (Luhn 1958 and Edmundson 1969), the field came to a virtual halt until the nineties. It is only then that a revival has taken place (see, for example, Mani and Maybury 1999; Mani 2001; Afantenos et al. 2005a for various overviews). Those early works were mostly concerned with the creation of text summaries from a single source. Multi-Document Summarization (MDS) wouldn't be actively pursued until after the mid-1990's - since when it is a quite active area of research.

Despite its youth, a consensus has emerged within the research community concerning the way to proceed in order to solve the problem. What seems to be at the core of MDS is the identification of similarities and differences between related documents (Mani and Bloedorn 1999; Mani 2001; see also EndresNiggemeyer 1998 and Afantenos et al. 2005a). This is generally translated as the identification of informationally equivalent passages in the texts. In order to achieve this goal/state, researchers use various methods ranging from statistical (Goldstein et al. 2000), to syntactic (Barzilay et al. 1999) or semantic approaches (Radev and McKeown 1998).

Despite this consensus, most researchers do not know precisely what they mean when they refer to these similarities or differences. What we propose here is that, at least for the problem at hand, i.e. of the summarization of evolving events, we should view the identification of the similarities and differences on two axes: the synchronic and diachronic axis. In the former case we are mostly concerned with the relative agreement of the various sources, within a given time frame, whilst in the latter case we are concerned with the actual evolution of an event, as it is being described by a single source.

Hence, in order to capture these similarities and differences we propose to use, what we call, the Synchronic and Diachronic Relations (henceforth SDRs) across the documents. The seeds of our SDRs lie of course in Mann and Thompson's $(1987,1988)$ Rhetorical Structure Theory (RST). While RST will be more thoroughly discussed in section 8 , let us simply mention here that it was initially developed in the context of "computational text generation", ${ }^{2}$ in order to relate a set of small text segments (usually clauses) into a larger, rhetorically motivated whole (text). The relations in charge of gluing the chunks (text segments) are semantic in nature, and they are supposed to capture the authors' (rhetorical) intentions, hence their name. ${ }^{3}$

\footnotetext{
${ }^{1}$ http://www.google.com/news

${ }^{2}$ Also referred to as Natural Language Generation (NLG).

${ }^{3}$ In fact, the opinions concerning what RST relations are supposed to represent, vary considerably. According to one view, they represent the author's intentions; while according
} 
Synchronic and Diachronic Relations (SDRs) are similar to RST relations in the sense that they are supposed to capture similarities and differences, i.e. the semantic relations, holding between conceptual chunks, of the input (documents), on the synchronic and diachronic axis. The question is, what are the units of analysis for the SDRs? Akin to work in NLG we could call these chunks "messages". Indeed, the initial motivation for SDRs was the belief or hope that the semantic information they carry could be exploited later on by a generator for the final creation of the summary.

In the following sections, we will try to clarify what messages and SDRs are, as well as provide some formal definitions. However, before doing so, we will present in section 2 a discussion concerning the nature of events, as well as a distinction between linearly and non-linearly evolving events. Section 3 provides a general overview of our approach, while section 4 contains an in-depth discussion of the Synchronic and Diachronic Relations. In sections 5 and 6 we present two concrete examples of systems we have built for the creation of Evolving Summaries in a linearly and non-linearly evolving topic. Section 7 provides a discussion concerning the relationship/relevance of our approach with a Natural Language Generation system, effectively showing how the computational extraction of the messages and SDRs can be considered as the first stage, out of three, of a typically pipelined NLG system. Section 8 presents related work, focusing on the link between our theory and Rhetorical Structure Theory. In section 9 we conclude, by presenting some thoughts concerning future research.

\section{Some Definitions}

This work is about the summarization of events that evolve through time. A natural question that can arise at this point is what is an event, and how do events evolve? Additionally, for a particular event, do all the sources follow its evolution or does each one have a different rate for emitting their reports, possibly aggregating several activities of the event into one report? Does this evolution of the events affect the summarization process?

Let us first begin by answering the question of "what is an event?" In the Topic Detection and Tracking (TDT) research, an event is described as "something that happens at some specific time and place" (Papka 1999, p 3; see also Allan et al. 1998a). The inherent notion of time is what distinguishes the event from the more general term topic. For example, the general class of terrorist incidents which include hostages is regarded as a topic, while a particular instance of this class, such as the one concerning the two Italian women that were kept as hostages by an Iraqi group in 2004, is regarded as an event. In general then, we can say that a topic is a class of events while an event is an instance of a particular topic.

An argument that has been raised in the TDT research is that although the definition of an event as "something that happens at some specific time and place" serves us well in most occasions, such a definition does have some

to another, they represent the effects they are supposed to have on the readers. The interested reader is strongly advised to take a look at the original papers by Mann and Thompson (1987, 1988), or at Taboada and Mann (2006). 
problems (Allan et al. 1998b). As an example, consider the occupation of the Moscow Theater in 2002 by Chechen extremists. Although this occupation spans several days, many would consider it as being a single event, even if it does not strictly happen at some "specific time". The consensus that seems to have been achieved among the researchers in TDT is that events indeed exhibit evolution, which might span a considerable amount of time (Papka 1999; Allan et al. 1998b). Cieri (2000), for example, defines an event to be as "a specific thing that happens at a specific time and place along with all necessary preconditions and unavoidable consequences", a definition which tries to reflect the evolution of an event.

Another distinction that the researchers in TDT make is that of the $a c$ tivities. An activity is "a connected set of actions that have a common focus or purpose" (Papka 1999, p 3). The notion of activities is best understood through an example. Take for instance the topic of terrorist incidents that involve hostages. A specific event that belongs to this topic is composed of a sequence of activities, which could, for example, be the fact that the terrorists have captured several hostages, the demands that the terrorists have, the negotiations, the fact that they have freed a hostage, etc. Casting a more close look on the definition of the activities, we will see that the activities are further decomposed into a sequence of more simple actions. For example, such actions for the activity of the negotiations can be the fact that a terrorist threatens to kill a specific hostage unless certain demands are fulfilled, the possible denial of the negotiation team to fulfil those demands and the proposition by them of something else, the freeing of a hostage, etc. In order to capture those actions, we use a structure which we call message - briefly mentioned in the introduction of this paper. In our discussion of topics, events and activities we will adopt the definitions provided by the TDT research.

Having thus provided a definition of topics, events and activities, let us now proceed with our next question of "how do events evolve through time". Concerning this question, we distinguish between two types of evolution: linear and non-linear. In linear evolution the major activities of an event are happening in predictable and possibly constant quanta of time. In non-linear evolution, in contrast, we cannot distinguish any meaningful pattern in the order that the major activities of an event are happening. This distinction is depicted in Figure 1 in which the evolution of two different events is depicted with the dark solid circles.

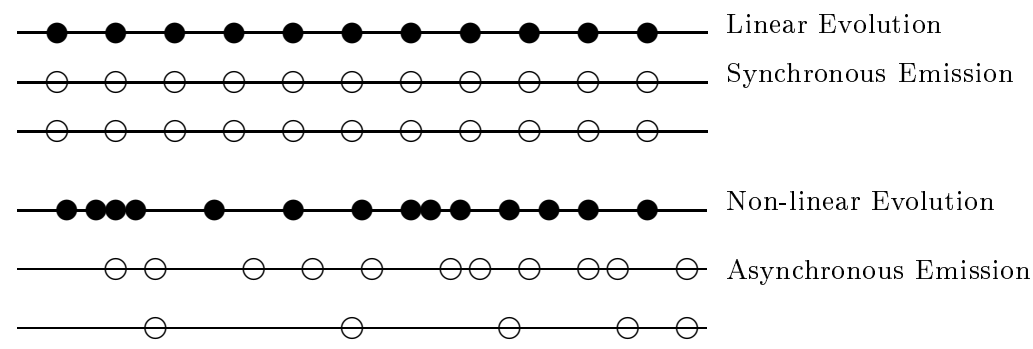

Figure 1: Linear and Non-linear evolution 
At this point we would like to formally describe the notion of linearity. As we have said, an event is composed of a series of activities. We will denote this as follows:

$$
E=\left\{a_{1}, a_{2}, \ldots, a_{n}\right\}
$$

where each activity $a_{i}$ occurs at a specific point in time, which we will denote as follows:

$$
\left|a_{i}\right|_{\text {time }}=t_{i}
$$

Such an event $E$ will exhibit linear evolution if

$$
\forall k \in\{2,3, \ldots, n\} \exists m \in \mathbb{N}:\left|a_{k}\right|_{\text {time }}-\left|a_{k-1}\right|_{\text {time }}=m \cdot t
$$

where $t$ is a constant time unit. On all other cases the event $E$ will exhibit non-linear evolution. As we have said, linearly evolving events reflect organized human actions that have a periodicity. Take for instance the event of a specific football championship. The various matches that compose such an event ${ }^{4}$ usually have a constant temporal distance between them. Nevertheless, it can be the case that a particular match might be canceled due, for example, to the holidays season, resulting thus in an "empty slot" in place of this match. Equation (1) captures exactly this phenomenon. Usually the value of $m$ will be 1 , having thus a constant temporal distance between the activities of an event. Occasionally though, $m$ can take higher values, e.g. 2, making thus the temporal distance between two consecutive activities twice as big as we would normally expect. In non-linearly evolving events, on the other hand, the activities of the events do not have to happen in discrete quanta of time; instead they can follow any conceivable pattern. Thus any event, whose activities do not follow the pattern captured in Equation (1), will exhibit non-linear evolution.

Linearly evolving events have a fair proportion in the world. They can range from descriptions of various athletic events to quarterly reports that an organization is publishing. In particular we have examined the descriptions of football matches (Afantenos et al. 2004; Afantenos et al. 2005b; see also section 5). On the other hand, one can argue that most of the events that we find in the news stories are non-linearly evolving events. They can vary from political ones, such as various international political issues, to airplane crashes or terrorist events. As a non-linearly evolving topic, we have investigated the topic of terrorist incidents which involve hostages (see section 6).

Coming now to the question concerning the rate with which the various sources emit their reports, we can distinguish between synchronous and asynchronous emission of reports. In the case of synchronous emission of reports, the sources publish almost simultaneously their reports, whilst in the case of asynchronous emission of reports, each source follows its own agenda in publishing their reports. This distinction is depicted in Figure 1 with the white circles. In most of the cases, when we have an event that evolves linearly we will also have a synchronous emission of reports, since the various sources can easily adjust to

\footnotetext{
${ }^{4}$ In this case, the topic is Football Championships, while a particular event could be the French football championship of 2005-2006. We consider each match to be an activity, since according to the definitions given by the TDT it constitutes "a connected set of actions that have a common focus or purpose".
} 
the pattern of the evolution of an event. This cannot be said for the case of non-linear evolution, resulting thus in asynchronous emission of reports by the various sources.

Having formally defined the notions of linearly and non-linearly evolving events, let us now try to formalize the notion of synchronicity as well. In order to do so, we will denote the description of the evolution of an event from a source $S_{i}$ as

$$
S_{i}=\left\{r_{i 1}, r_{i 2}, \ldots r_{i n}\right\}
$$

or more compactly as

$$
S_{i}=\left\{r_{i j}\right\}_{j=1}^{n}
$$

where each $r_{i j}$ represents the $j$ th report from source $S_{i}$. Each $r_{i j}$ is accompanied by its publication time which we will denote as

$$
\left|r_{i j}\right|_{\text {pub_time }}
$$

Now, let us assume that we have two sources $S_{k}$ and $S_{l}$ which describe the same event, i.e.

$$
\begin{aligned}
S_{k} & =\left\{r_{k i}\right\}_{i=1}^{n} \\
S_{l} & =\left\{r_{l i}\right\}_{i=1}^{m}
\end{aligned}
$$

This event will exhibit a synchronous emission of reports if and only if

$$
\begin{gathered}
m=n \quad \text { and } \\
\forall i:\left|r_{k i}\right|_{\text {pub_time }}=\left|r_{l i}\right|_{\text {pub_time }}
\end{gathered}
$$

Equation (3) implies that the two sources have exactly the same number of reports, while Equation (4) implies that all the corresponding reports are published simultaneously. On the other hand, the event will exhibit non-linear evolution with asynchronous emission of reports if and only if

$$
\exists i:\left|r_{k i}\right|_{\text {pub_time }} \neq\left|r_{l i}\right|_{\text {pub_time }}
$$

Equation (5) implies that at least two of the corresponding reports of $S_{k}$ and $S_{l}$ have a different publication time. Usually of course, we will have more than two reports that will have a different publication time. Additionally we would like to note that the $m$ and $n$ of (2) are not related, i.e. they might or might not be equal. ${ }^{5}$

In Figure 2 we represent two events which evolve linearly and non-linearly and for which the sources report synchronously and asynchronously respectively. The vertical axes in this figure represent the number of reports per source on a particular event. The horizontal axes represents the time, in weeks and days respectively, that the documents are published. The first event concerns descriptions of football matches. In this particular event we have constant reports weekly from 3 different sources for a period of 30 weeks. The lines for each source

\footnotetext{
${ }^{5}$ In the formal definitions that we have provided for the linear and non-linear evolution of the events, as well as for the synchronous and asynchronous emission of reports, we have focused in the case that we have two sources. The above are easily extended for cases where we have more than two sources.
} 
fall on top of each other since they publish simultaneously. The second event concerns a terrorist group in Iraq which kept as hostages two Italian women. In the figure we depict 5 sources. The number of reports that each source is making varies from five to twelve, in a period of about 23 days. As we can see from the figure, most of the sources begin reporting almost instantaneously, except one which delays its report for about twelve days. Another source, although it reports almost immediately, it delays considerably subsequent reports.
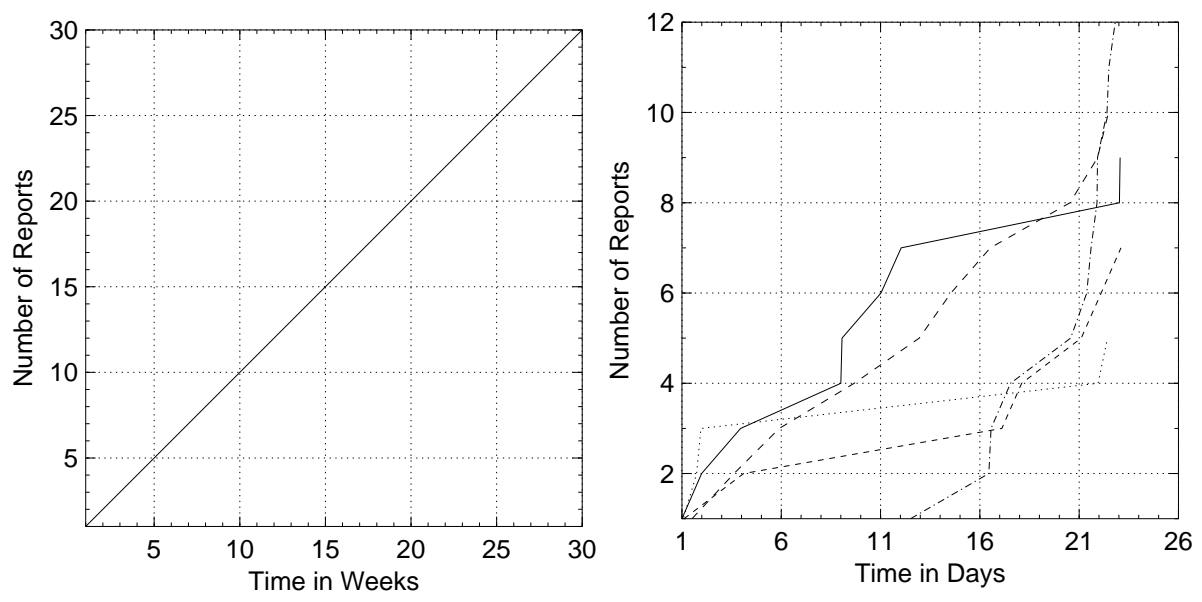

Figure 2: Linear and Non-linear evolution

Let us now come to our final question, namely whether the linearity of an event and the synchronicity of the emission of reports affects our summarization approach. As it might have been evident thus far, in the case of linear evolution with synchronous emission of reports, the reports published by the various sources which describe the evolution of an event, are well aligned in time. In other words, time in this case proceeds in quanta and in each quantum each source emits a report. This has the implication that, when the final summary is created, it is natural that the NLG component that will create the text of the summary (see sections 3 and 7 ) will proceed by "summarizing" 6 each quantum - i.e. the reports that have been published in this quantum - separately, exploiting firstly the Synchronic relations for the identification of the similarities and differences that exist synchronically for this quantum. At the next step, the NLG component will exploit the Diachronic relations for the summarization of the similarities and differences that exist between the quanta - i.e. the reports published therein - showing thus the evolution of the event.

In the case though of non-linear evolution with asynchronous emission of reports, time does not proceed in quanta, and of course the reports from the various sources are not aligned in time. Instead, the activities of an event can follow any conceivable pattern and each source can follow its own agenda on publishing the reports describing the evolution of an event. This has two

\footnotetext{
${ }^{6}$ The word "summarizing" here ought to be interpreted as the Aggregation stage in a typical architecture of an NLG system. See section 7 for more information on how our approach is related to NLG.
} 
implications. The first is that, when a source is publishing a report, it is very often the case that it contains the description of many activities that happened quite back in time, in relation always to the publication time of the report. This is best viewed in the second part of Figure 2. As you can see in this figure, it can be the case that a particular source might delay the publication of several activities, effectively thus including the description of various activities into one report. This means that several of the messages included in such reports will refer to a point in time which is different from their publication time. Thus, in order to connect the messages with the Synchronic and Diachronic Relations the messages ought to be placed first in their appropriate point in time in which they refer. ${ }^{7}$ The second important implication is that, since there is no meaningful quantum of time in which the activities happen, then the summarization process should proceed differently from the one in the case of linear evolution. In other words, while in the first case the Aggregation stage of the NLG component (see section 7) can take into account the quanta of time, in this case it cannot, since there are no quanta in time in which the reports are aligned. Instead the Aggregation stage of the NLG component should proceed differently. Thus we can see that our summarization approach is indeed affected by the linearity of the topic.

\section{A General Overview}

As we have said in the introduction of this paper, the aim of this study is to present a methodology for the automatic creation of summaries from evolving events. Our methodology is composed of two main phases, the topic analysis phase and the implementation phase. The first phase aims at providing the necessary domain knowledge to the system, which is basically expressed through an ontology and the specifications of the messages and the SDRs. The aim of the second phase is to locate in the text the instances of the ontology concepts, the messages and the SDRs, ultimately creating a structure which we call the grid. The creation of the grid constitutes, in fact, the first stage - the Document Planning - out of the three typical stages of an NLG system (see section 7 for more details). The topic analysis phase, as well as the training of the summarization system, is performed once for every topic, and then the system is able to create summaries for each new event that is an instance of this topic. In this section we will elaborate on those two phases, and present the general architecture of a system for creating summaries from evolving events. During the examination of the topic analysis phase we will also provide a brief introduction of the notions of SDRs, which we more thoroughly present in section 4 . An in-depth examination on the nature of messages is presented in section 3.1.2.

\subsection{Topic Analysis Phase}

The topic analysis phase is composed of four steps, which include the creation of the ontology for the topic, the providing of the specifications for the messages

\footnotetext{
${ }^{7}$ It could be the case that, even for the linearly evolving events, some sources might contain in their reports small descriptions of prior activities from the ones in focus. Although we believe that such a thing is rare, it is the responsibility of the system to detect such references and handle appropriately the messages. In the case-study of a linearly evolving event (section 5) we did not identify any such cases.
} 
and the Synchronic and Diachronic Relations. The final step of this phase, which in fact serves as a bridge step with the implementation phase, includes the annotation of the corpora belonging to the topic under examination that have to be collected as a preliminary step during this phase. The annotated corpora will serve a dual role: the first is the training of the various Machine Learning algorithms used during the next phase and the second is for evaluation purposes (see sections 5 and 6 ). In the following we will describe in more detail the four steps of this phase. A more thorough examination of the Synchronic and Diachronic Relations is presented in section 4.

\subsubsection{Ontology}

The first step in the topic analysis phase is the creation of the ontology for the topic under focus. Ontology building is a field which, during the last decade, not only has gained tremendous significance for the building of various natural language processing systems, but also has experienced a rapid evolution. Despite that evolution, a converged consensus seems to have been achieved concerning the stages involved in the creation of an ontology (Pinto and Martins 2004; Jones et al. 1998; Lopez 1999). Those stages include the specification, the conceptualization, the formalization and the implementation of the ontology. The aim of the first stage involves the specification of the purpose for which the ontology is built, effectively thus restricting the various conceptual models used for modeling, i.e. conceptualizing, the domain. The conceptualization stage includes the enumeration of the terms that represent concepts, as well as their attributes and relations, with the aim of creating the conceptual description of the ontology. During the third stage, that conceptual description is transformed into a formal model, through the use of axioms that restrict the possible interpretations for the meaning of the formalized concepts, as well as through the use of relations which organize those concepts; such relations can be, for example, is-a or part-of relations. The final stage concerns the implementation of the formalized ontology using a knowledge-representation language. ${ }^{8}$ In the two case-studies of a linearly and non-linearly evolving topic, which we present in sections 5 and 6 respectively, we follow those formal guidelines for the creation of the ontologies.

\subsubsection{Messages}

Having provided an ontology for the topic, the next step in our methodology is the creation of the specifications for the messages, which represent the actions involved in a topic's events. In order to define what an action is about, we have to provide a name for the message that represents that action. Additionally, each action usually involves a certain number of entities. The second step, thus, is to associate each message with the particular entities that are involved in the action that this message represents. The entities are of course taken from the formal definition of the ontology that we provided in the previous step. Thus, a message

\footnotetext{
${ }^{8}$ In fact, a fifth stage exists, as well, for the building of the ontology, namely that of maintenance, which involves the periodic update and correction of the implemented ontology, in terms of adding new variants of new instances to the concepts that belong to it, as well as its enrichment, i.e. the addition of new concepts. At the current state of our research, this step is not included; nevertheless, see the discussion in section 9 on how this step can, in the future, enhance our approach.
} 
is composed of two parts: its name and a list of arguments which represent the ontology concepts involved in the action that the message represents. Each argument can take as value the instances of a particular ontology concept or concepts, according to the message definition. Of course, we shouldn't forget that a particular action is being described by a specific source and it refers to a specific point in time. Thus the notion of time and source should also be incorporated into the notion of messages. The source tag of a message is inherited from the source which published the document that contains the message. If we have a message $m$, we will denote the source tag of the message as $|m|_{\text {source }}$. Concerning the time tag, this is divided into two parts: the publication time which denotes the time that the document which contains the message was published, and the referring time which denotes the actual time that the message refers to. The message's publication time is inherited from the publication time of the document in which it is contained. The referring time of a message is, initially, set to the publication time of the message, unless some temporal expressions are found in the text that alter the time to which the message refers. The publication and referring time for a message $m$ will be denoted as $|m|_{\text {pub_time }}$ and $|m|_{\text {ref_time }}$ respectively. Thus, a message can be defined as follows. ${ }^{9}$

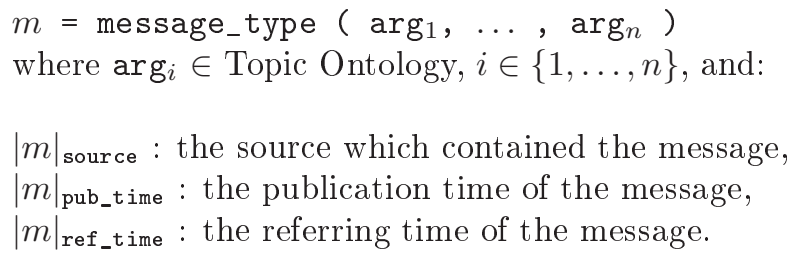

A simple example might be useful at this point. Take for instance the case of the hijacking of an airplane by terrorists. In such a case, we are interested in knowing if the airplane has arrived to its destination, or even to another place. This action can be captured by a message of type arrive whose arguments can be the entity that arrives (the airplane in our case, or a vehicle, in general) and the location that it arrives. The specifications of such a message can be expressed as follows:

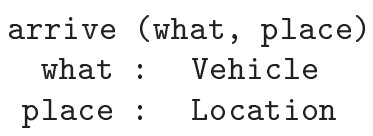

The concepts Vehicle and Location belong to the ontology of the topic; the concept Airplane is a sub-concept of the Vehicle. A sentence that might instantiate this message is the following:

The Boeing 747 arrived yesterday at the airport of Stanstend.

For the purposes of this example, we will assume that this sentence was emitted from source $A$ on 12 February, 2006. The instance of the message is

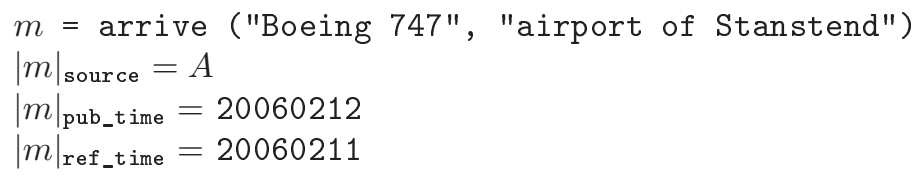

${ }^{9}$ See also (Afantenos et al. 2004; Afantenos et al. 2005b; Afantenos et al. 2005c). 
As we can see, the referring time is normalized to one day before the publication of the report that contained this message, due to the appearance of the word "yesterday" in the sentence.

The role of the messages' referring time-stamp is to place the message in the appropriate time-frame, which is extremely useful when we try to determine the instances of the Synchronic and Diachronic Relations. Take a look again at the second part of Figure 2. As you can see from that figure, there is a source that delays considerably the publication of its first report on the event. Inevitably, this first report will try to brief up its readers with the evolution of the event thus far. This implies that it will mention several activities of the event that will not refer to the publication time of the report but much earlier, using, of course, temporal expressions to accomplish this. The same happens with another source in which we see a delay between the sixth and seventh report.

At this point, we have to stress that the aim of this step is to provide the specifications of the messages, which include the provision of the message types as well as the list of arguments for each message type. This is achieved by studying the corpus that has been initially collected, taking of course into consideration the ontology of the topic as well. The actual extraction of the messages' instances, as well as their referring time, will be performed by the system which will be built during the next phase. Additionally, we would like to note that our notion of messages are similar structures (although simpler ones) to the templates used in the Message Understanding Conferences (MUC). ${ }^{10}$

\subsubsection{Synchronic and Diachronic Relations}

Once we have provided the specifications of the messages, the next step in our methodology is to provide the specifications of the Synchronic and Diachronic Relations, which will connect the messages across the documents. Synchronic relations connect messages from different sources that refer $^{11}$ to the same time frame, while Diachronic relations connect messages from the same source, but which refer to different time frames. SDRs are not domain dependent relations, which implies that they are defined for each topic. In order to define a relation we have to provide a name for it, which carries semantic information, and describes the conditions under which this relation holds, taking into consideration the specifications of the messages. For example, if we have two different arrive messages

$$
\begin{aligned}
& m_{1}=\operatorname{arrive}\left(\text { vehicle }_{1}, \text { location }_{1}\right) \\
& \left.m_{2}=\text { arrive (vehicle } 2, \text { location }_{2}\right)
\end{aligned}
$$

and they belong to different sources (i.e. $\left.\left|m_{1}\right|_{\text {source }} \neq\left|m_{2}\right|_{\text {source }}\right)$ but refer to the same time frame (i.e. $\left.\left|m_{1}\right|_{\text {ref_time }}=\left|m_{2}\right|_{\text {ref_time }}\right)$ then they will be connected with the Disagreement Synchronic relation if:

$$
\begin{aligned}
& \text { vehicle }_{1}=\text { vehicle }_{2} \text { and } \\
& \text { location }_{1} \neq \text { location }_{2}
\end{aligned}
$$

On the other hand, if the messages belong to the same source (i.e. $\left|m_{1}\right|_{\text {source }}=$ $\left.\left|m_{2}\right|_{\text {source }}\right)$, but refer to different time frames (i.e. $\left.\left|m_{1}\right|_{\text {ref_time }} \neq\left|m_{2}\right|_{\text {ref_time }}\right)$, they will be connected with the Repetition Diachronic relation if:

\footnotetext{
${ }^{10}$ http://www-nlpir.nist.gov/related_projects/muc/proceedings/muc_7_toc.html

${ }^{11}$ What we mean by the use of the word "refer" here is that in order to connect two messages with an SDR we are using their referring time instead of their publication time.
} 


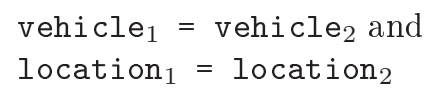

Synchronic and Diachronic Relations are more thoroughly examined in section 4 .

\subsubsection{Corpora Annotation}

The fourth and final step in our methodology is the annotation of the corpora, which ought to have been collected as a preliminary step of this phase. In fact, this step can be viewed as a "bridge step" with the next phase - the implementation phase - since the information that will be annotated during this step, will be used later in that phase for the training of the various Machine Learning algorithms, as well as for the evaluation process. In essence, we annotate three kinds of information during this step. The first is the entities which represent the ontology concepts. We annotate those entities with the appropriate ontology (sub)concepts. The next piece of information that we have to annotate is the messages. This annotation process is in fact split into two parts. In the first part we have to annotate the textual elements of the input documents which represent the message types. In the second part we have to connect those message types with their corresponding arguments. In most of the cases, as we also mention in sections 5 and 6 , we will have an one-to-one mapping from sentences to message types, which implies that we will annotate the sentences of the input documents with the appropriate message type. In the second part we will connect those message types with their arguments, which are in essence the entities previously annotated. Those entities are usually found in the sentence under consideration or in the near vicinity of that sentence. Finally we will have to annotate the SDRs as well. This is performed by applying the rules provided in the specification of the Relations (see also section 4) to the previously annotated messages. The annotation of the entities, messages and SDRs provides us with a "gold corpus" which will be used for the training of the various Machine Learning algorithms as well as for the evaluation process.

\subsection{Implementation Phase}

The topic analysis phase is performed once for each topic, ${ }^{12}$ so that the necessary domain knowledge will be provided to the summarization system which will produce the summaries for each new event that belongs to this topic. The core of the summarization system is depicted in Figure 3. As you can see, this system takes as input a set of documents related to the event that we want to summarize. Those documents, apart from their text, contain two additional pieces of information: their source and their publication time. This information will be used for the determination of the source and publication/referring time of the messages that are contained in each document. The system is composed of four main stages. In this section we will briefly mention what the role of each stage is, providing some clues on the possible computational approaches that can be used. In sections 5 and 6 we will present two concrete computational implementations for a linearly and a non-linearly evolving topic.

\footnotetext{
${ }^{12}$ Although this is certainly true, in section 9 we provide a discussion on how the system might cope with novel concepts that might arise in new events that belong to a topic and which have not been included in the originally created ontology. This discussion is also extended for the case of messages.
} 


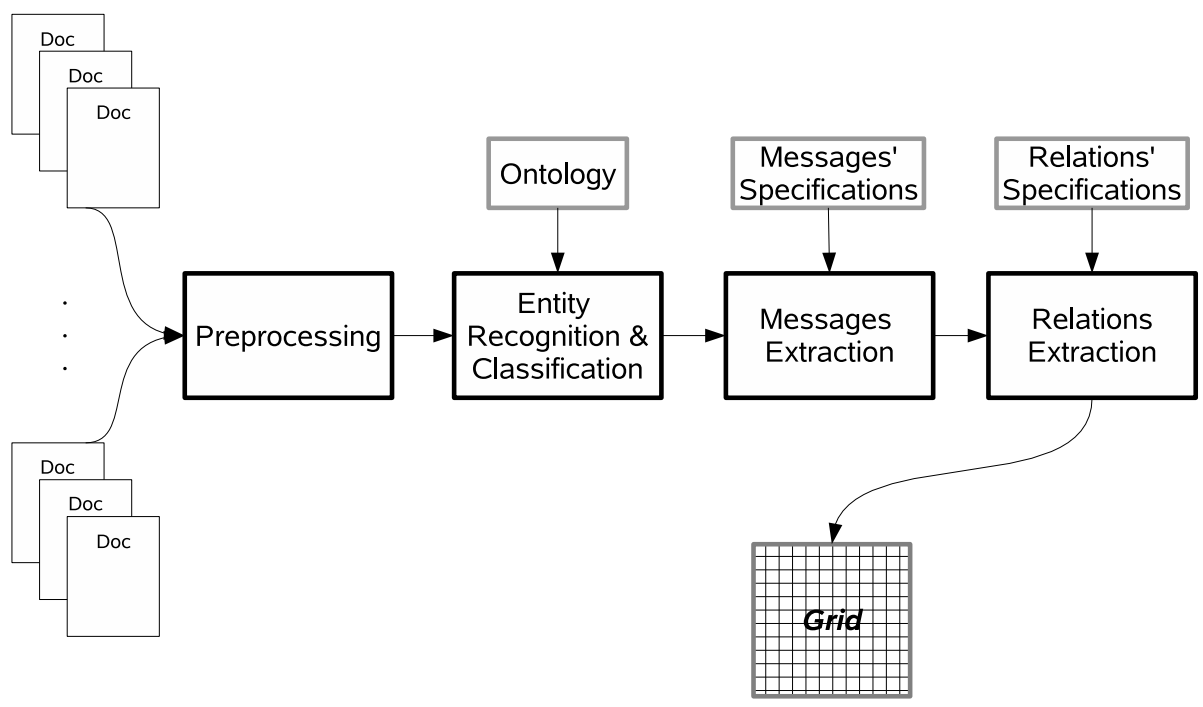

Figure 3: The summarization system.

The first stage of the system is a preprocessing that we perform in the input documents. This preprocessing may vary according to the topic, and it is actually driven by the needs that have the various Machine Learning algorithms which will be used in the following stages. In general, this stage is composed of modules such as a tokenizer, a sentence splitter, a part-of-speech tagger etc. For example, in the vast majority of cases (as we explain in sections 5 and 6) we had an one-to-one mapping of sentences to messages. Thus, a sentence splitter is needed in order to split the document into sentences that will be later classified into message types. The actual Machine Learning algorithms used will be presented in sections 5 and 6 .

The next stage of the system is the Entities Recognition and Classification stage. This stage takes as input the ontology of the topic, specified during the previous phase, and its aim is to identify the textual elements in the input documents which denote the various entities, as well as to classify them in their appropriate (sub)concepts, according to the ontology. The methods used in order to tackle that problem vary. If, for example, the entities and their textual realizations are a priori known, then the use of simple gazetteers might suffice. In general though, we wouldn't normally expect something similar to happen. Thus, a more complex process, usually including Machine Learning ought to be used for this stage. The identified entities will later be used for the filling in of the messages' arguments.

The third stage is concerned with the extraction of the messages from the input documents. The aim of this stage is threefold, in fact. The first thing that should be done is the mapping of the sentences in the input documents to message types. In the two case studies that we have performed, and which are more thoroughly described in sections 5 and 6 , we came to the conclusion that in most of the cases, as mentioned earlier, we have an one-to-one mapping from sentences to message types. In order to perform the mapping, we are training 
Machine Learning based classifiers. In sections 5 and 6 we will provide the full details for the two particular topics that we have studied. The next thing that should be performed during this stage is the filling in of the messages' arguments; in other words, the connection of the entities identified in the previous stage with the message types. We should note that, in contrast with the mapping of the sentences to message types, in this case we might find several of the messages' arguments occurring in previous or even following sentences, from the ones under consideration. So, whatever methods used in this stage, they should take into account not only the sentences themselves, but their vicinity as well, in order to fill in the messages' arguments. The final task that should be performed is the identification of the temporal expressions in the documents that alter the referring time of the messages. The referring time should be normalized in relation to the publication time. Note that the publication time and the source tags of the messages are inherited from the documents which contain the messages.

The final stage in the summarization system is the extraction of the Synchronic and Diachronic Relations connecting the messages. This stage takes as input the relations' specifications and "interprets" them into an algorithm which takes as input the extracted messages, along with their source and publication/referring time which are attached to the messages. Then this algorithm is applied to the extracted messages from the previous stage, in order to identify the SDRs that connect them. The result of the above stages, as you can see in Figure 3 will be the creation of the structure that we have called grid.
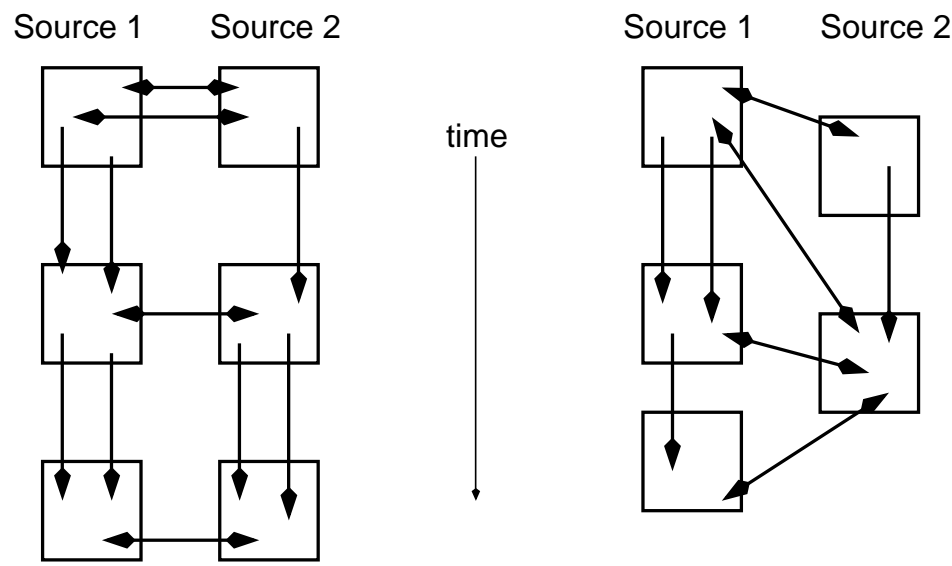

Figure 4: The grid structure with Synchronic and Diachronic relations for linearly and non-linearly evolving events.

The grid is a structure which virtually provides a level of abstraction over the textual information of the input documents. In essence, the grid is composed of the extracted messages, as well as the Synchronic and Diachronic Relations that connect them. A graphical representation of two grids, for a linearly evolving event with synchronous emission of reports and for a non-linearly evolving event with asynchronous emission of reports respectively, can be seen in Figure 4. In this figure the squares represent the documents that the sources emit, while 
the arrows represent the Synchronic and Diachronic Relations that connect the messages which are found inside the documents. In both cases, Synchronic relations connect messages that belong in the same time-frame, ${ }^{13}$ but in different sources, while Diachronic relations connect messages from different time-frames, but which belong in the same source. Although this is quite evident for the case of linear evolution, it merits some explanation for the case of non-linear evolution. As we can see in the second part of Figure 4, the Synchronic relations can connect messages that belong in documents from different time-frames. Nevertheless, as we have also mentioned in section 3.1 in order to connect two messages with an SDR we take into account their referring time instead of their publication time. In the case of linear evolution it is quite a prevalent phenomenon that the publication and referring time of the messages will be the same, making thus the Synchronic relations neatly aligned on the same timeframe. In the case, though, of non-linear evolution this phenomenon is not so prevalent, i.e. it is often the case that the publication and referring time of the messages do not coincide. ${ }^{14}$ This has the consequence that several of the Synchronic relations will look as if they connect messages which belong in different time-frames. Nevertheless, if we do examine the referring time of the messages, we will see that indeed they belong in the same time-frame.

As we have said, the grid provides a level of abstraction over the textual information contained in the input documents, in the sense that only the messages and relations are retained in the grid, while all the textual elements from the input documents are not being included. The creation of the grid constitutes, in essence, the first stage, the Document Planning, out of the three total stages in a typical NLG architecture (Reiter and Dale 2000). We would like to emphasize here the dynamic nature of the grid, concerning on-going events. It could be the case that the system can take as input a set of documents, from various sources, describing the evolution of an event up to a specific point in time. In such cases, the system will build a grid which will reflect the evolution of an event up to this point. Once new documents are given as input to the system, then the grid will be expanded by including the messages extracted from the new documents, as well as the SDRs that connect those messages with the previous ones or between them. Thus, the grid itself will evolve through time, as new documents are coming as input to the system, and accordingly the generated summary as well. The connection of the grid with the NLG is more thoroughly discussed in section 7 .

Finally this NLG system might as well, optionally, take as input a query from the user, the interpretation of which will create a sub-grid of the original grid. In this case, the sub-grid, instead of the original grid, will be summarized, i.e. will be transformed into a textual summary. In case that the user enters a query, then a query-based summary will be created, otherwise a generic one, capturing the whole evolution of the event, will be created. ${ }^{15}$

\footnotetext{
${ }^{13}$ A discussion of what we mean by the "same time-frame" can be found in section 4 . For the moment, suffice it to say that the same time frame can vary, depending on the topic. In sections 5 and 6 we provide more details for the choices we have made for two different case studies.

${ }^{14}$ If we cast a look again at the second part of Figure 2 we will see why this is the case. As we can see there, several sources delay the publication of their reports. This implies that they can provide information on several of the past activities of the events, making thus the messages to have different publication and referring times.

${ }^{15} \mathrm{On}$ the distinction between generic and query-based summaries see Afantenos et al. (2005a,
} 


\section{Synchronic and Diachronic Relations}

The quintessential task in the Multi-Document Summarization research, as we have already mentioned in the introduction of this paper, is the identification of similarities and differences between the documents. Usually, when we have the first activity of an event happening, there will be many sources that will commence describing that event. It is obvious that the information the various sources have at this point will vary, leading thus to agreements and contradictions between them. As the event evolves, we will possibly have a convergence on the opinions, save maybe for the subjective ones. We believe that the task of creating a summary for the evolution of an event entails the description of its evolution, as well as the designation of the points of confliction or agreement between the sources, as the event evolves. In order to capture the evolution of an event as well as the conflict, agreement or variation between the sources, we introduce the notion of Synchronic and Diachronic Relations. Synchronic relations try to identify the degree of agreement, disagreement or variation between the various sources, at about the same time frame. Diachronic relations, on the other hand, try to capture the evolution of an event as it is being described by one source.

According to our viewpoint, Synchronic and Diachronic Relations ought to be topic-dependent. To put it differently, we believe that a "universal" taxonomy of relations, so to speak, will not be able to fulfil the intricacies and needs, in terms of expressive power, ${ }^{16}$ for every possible topic. Accordingly, we believe that SDRs ought to be defined for each new topic, during what we have called in section 3 the "topic analysis" phase. We would like though to caution the reader that such a belief does not imply that a small pool of relations which are independent of topic, such as for example Agreement, Disagreement or Elaboration, could not possibly exist. In the general case though, SDRs are topic-dependent.

As we have briefly mentioned in the introduction of this paper, Synchronic and Diachronic Relations hold between two different messages. More formally, a relation definition consists of the following four fields:

1. The relation's type (i.e. Synchronic or Diachronic).

2. The relation's name.

3. The set of pairs of message types that are involved in the relation.

4. The constraints that the corresponding arguments of each of the pairs of message types should have. Those constraints are expressed using the notation of first order logic.

The name of the relation carries semantic information which, along with the messages that are connected with the relation, are later being exploited by the Natural Language Generation component (see section 7) in order to produce the final summary. Following the example of subsection 3.1, we would formally define the relations Disagreement and Repetition as shown in Table 1.

\footnotetext{
p 159).

${ }^{16}$ We are talking about the "expressive power" of an SDR, since SDRs are ultimately passed over to an NLG system, in order to be expressed in a natural language.
} 


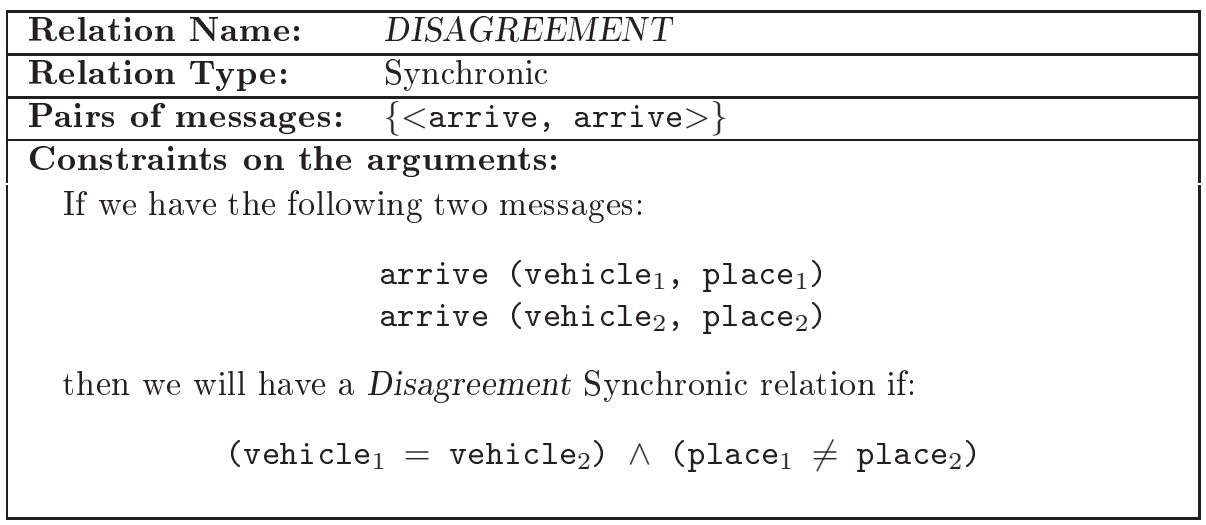

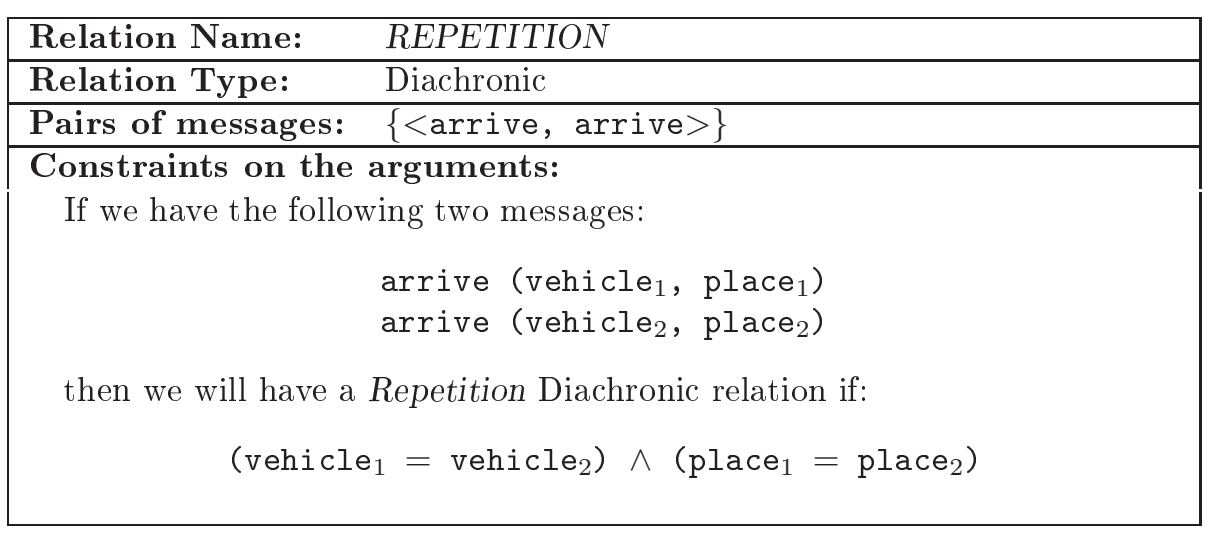

Table 1: Example of formal definitions for two relations.

The aim of the Synchronic relations is to capture the degree of agreement, disagreement or variation that the various sources have for the same time-frame. In order thus to define the Synchronic relations, for a particular topic, the messages that they connect should belong to different sources, but refer to the same time-frame. A question that naturally arises at this point is, what do we consider as the same time-frame? In the case of a linearly evolving event with a synchronous emission of reports, this is an easy question. Since all the sources emit their reports in constant quanta of time, i.e. at about the same time, we can consider each emission of reports by the sources, as constituting an appropriate time-frame. This is not though the case in an event that evolves non-linearly and exhibits asynchronicity in the emission of the reports. As we have discussed in section 3, in such cases, several of the messages will have a reference in time that is different from the publication time of the document that contains the message. In such cases we should impose a time window, in relation to the referring time of the messages, within which all the messages can be considered as candidates for a connection with a synchronic relation. This time window can vary from several hours to some days, depending on the topic and the rate with which the sources emit their reports. In sections 5 and 6 , where we present two case-studies on a linearly and a non-linearly evolving topics respectively, we will more thoroughly present the choices that we have made in relation to the time window. 
The aim of Diachronic relations, on the other hand, is to capture the evolution of an event as it is being described by one source. In this sense then Diachronic relations do not exhibit the same challenges that the Synchronic ones have, in relation to time. As candidate messages to be connected with a Diachronic relation we can initially consider all the messages that belong to the same source but have a different referring time - but not the same publication time since that implies that the messages belong in the same document, something that would make our relations intra-document, instead of cross-document, as they are intended.

A question that could arise at this point, concerns the chronological distance that two messages should have in order to be considered as candidates for a connection with a Diachronic relation. The distance should, definitely, be more than zero, i.e. the messages should not belong in the same time frame. But, how long could the chronological distance be? It turns out that it all depends on the topic, and the time that the evolution of the event spans. Essentially, the chronological distance in which two messages should be considered as candidates for a connection with a Diachronic relation, depends on the distance in time that we expect the actions of the entities to affect later actions. If the effects are expected to have a "local" temporal effect, then we should opt for a small chronological distance, otherwise we should opt for a long one. In the case-study for the linearly evolving topic (section 5), we chose to have a small temporal distance, whilst in the non-linearly evolving topic (section 6), we chose to have no limit on the distance. ${ }^{17}$ The reason for those decisions will become apparent on the respective sections.

Until now, in our discussion of the Synchronic and Diachronic Relations, we have mainly concentrated on the role that the source and time play, in order for two messages to be considered as candidates for a connection with either a Synchronic or a Diachronic relation. In order though to establish an actual relation between two candidate messages, we should further examine the messages, by taking into account their types and their arguments. In other words, in order to establish a relation we should provide some rules that take into account the messages' types as well as the values of their arguments. In most of the cases, we will have a relation between two messages that have the same message type, but this is not restrictive. In fact, in the non-linearly evolving topic that we have examined (section 6) we have defined several Diachronic relations that hold between different types of messages.

Once we have defined the names of the relations and their type, Synchronic or Diachronic, as well as the message pairs for which they hold, then for each relation we should describe the conditions that the messages should exhibit. Those conditions take into account the values that the messages' arguments have. Since the messages' arguments take their values from the topic ontology, those rules take into account the actual entities involved in the particular messages. Examples of such rules are provided in sections 5 and 6 .

\footnotetext{
${ }^{17}$ Of course, it should be greater than zero, otherwise a zero distance would make the relation Synchronic, not Diachronic.
} 


\section{Case Study I: Linear Evolution}

This section presents a case study which examines how our approach is applied to a linearly evolving topic, namely that of the descriptions of football matches. The reason for choosing this topic is that it is a rather not so complex one, which makes it quite ideal as a first test bed of our approach. This is a linearly evolving topic, since football matches occur normally once a week. Additionally, each match is described by many sources after the match has terminated, virtually at the same time. Thus we can consider that this topic exhibits synchronicity on the reports from the various sources. The linearity of the topic and synchronous emission of reports is depicted in the first part of Figure 2 (page 7), where we have the description of football matches from three sources for a period of 30 weeks. The lines from the three sources fall on top of each other reflecting the linearity and synchronicity of the topic.

\subsection{Topic Analysis}

The aim of the topic analysis phase, as we have thoroughly analyzed in section 3.1, is to collect an initial corpus for analysis, create the ontology for the topic and create the specifications for the messages and the relations, as well as the annotation of the corpus.

\subsubsection{Corpus Collection}

We manually collected descriptions of football matches, from three sources, for the period 2002-2003 of the Greek football championship. The sources we used were a newspaper ( $\mathrm{Ta} N e a$, http://digital.tanea.gr), a web portal (Flash, www.flash.gr) and the site of one football team (AEK, ww. aek.gr). The language used in the documents was Greek. This championship contained 30 rounds. We focused on the matches of a certain team, which were described by three sources. So, in total we collected 90 documents containing 64265 words.

\subsubsection{Ontology Creation}

After studying the collected corpus we created the ontology of the topic, following the formal guidelines in the field of ontology building, a summary of which we have presented in section 3.1. The concepts of the implemented ontology are connected with is-a relations. An excerpt of the final ontology can be seen in Figure 5 .

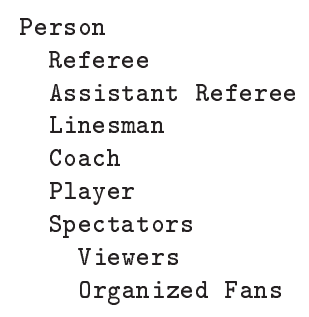

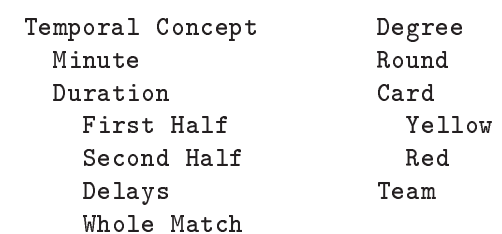

Whole Match

Figure 5: An excerpt from the topic ontology for the linearly evolving topic 


\subsubsection{Messages' Specifications}

Once we have defined the topic ontology, the next stage is the definition of the messages' specifications. This process includes two things: defining the message types that exist in the topic, as well as providing their full specifications. We concentrated in the most important actions, that is on actions that reflected the evolution of - for example - the performance of a player, or in actions that a user would be interested in knowing. At the end of this process we concluded on a set of 23 message types (Table 2). An example of full message specifications is shown in Figure 6. As you can see the arguments of the messages take their values from the topic ontology.

\begin{tabular}{|l|l|l|l|l|}
\hline Absent & Behavior & Block & Card & Goal_Cancelation \\
\hline Comeback & Final_Score & Foul & Injured & System_Selection \\
\hline Performance & Refereeship & Scorer & Change & Satisfaction \\
\hline Superior & Conditions & Penalty & Win & Opportunity_Lost \\
\hline Expectations & Hope_For & \multicolumn{2}{|c|}{ Successive_Victories } & \\
\hline
\end{tabular}

Table 2: Message types for the linearly evolving topic.

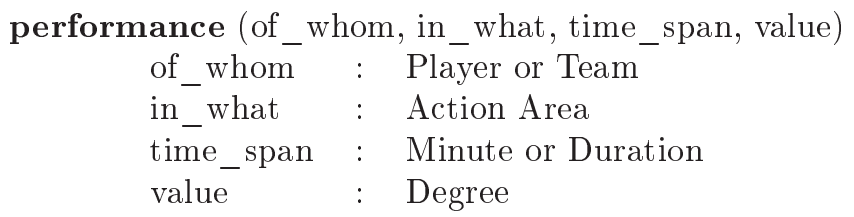

Figure 6: An example of message specifications for the linearly evolving topic.

\subsubsection{Relations' Specifications}

We concluded on twelve cross-document relations, six on the synchronic and six on the diachronic level (Table 3). Since this was a pilot-study during which we examined mostly the viability of our methodology, we limited the study of the cross-document relations, in relations that connect the same message types. Furthermore, concerning the Diachronic relations, we limited our study to relations that have chronological distance only one, where one corresponds to one week. ${ }^{18}$ Examples of such specifications for the message type performance are shown in Figure 7 . In the non-linearly evolving topic, examined in the following section, we have relations that connect different message types, and we impose no limits on the temporal distance that the messages should have in order to be connected with a Diachronic relation.

Having provided the topic ontology and the specifications of the messages and relations, we proceeded with the annotation of the corpora, as explained in section 3.1. We would like to add that the total amount of time required for the topic analysis phase was six months for a part-time work of two people.

\footnotetext{
${ }^{18}$ Chronological distance zero makes the relations synchronic.
} 


\begin{tabular}{|c|c|}
\hline Diachronic Relations & Synchronic Relations \\
\hline - Positive Graduation & - AGREEMENT \\
\hline - Negative Graduation & - Near Agreement \\
\hline - Stability & - Disagreement \\
\hline - Repetition & - Elaboration \\
\hline - Continuation & - Generalization \\
\hline - Generalizhtion & - PRECISEnESS \\
\hline
\end{tabular}

Table 3: Synchronic and Diachronic Relations in the linearly evolving topic

\subsection{Implementation}

This phase includes the identification in the input documents of the textual elements that represent ontology concepts, their classification to the appropriate ontology concept, as well as the computational extraction of the messages and Synchronic and Diachronic Relations. At the end of this process, the grid will be created, which in essence constitutes the Document Planning stage, the first out of three of a typical NLG architecture (see section 7). Casting a look again in Figure 3, we can see that the computational extraction of the grid consists of four stages. In the remaining of this subsection we will discuss those stages.

\subsubsection{Preprocessing}

The preprocessing stage is quite a simple one. It consists of a tokenization and a sentence splitting components. The information yielded from this stage will be used in the Entities Recognition and Classification stage and the messages' extraction stage. We would like to note that in order to perform this stage, as well as the following two, we used the ELLOGON platform (Petasis et al. 2002). ${ }^{19}$

\subsubsection{Entities Recognition and Classification}

As we discuss on section 3.2, the complexity of the Entities Recognition and Classification task can vary, depending on the topic. In the football topic this task was quite straightforward since all the entities involved in this topic, such as players and teams, were already known. Thus the use of simple gazetteer lists sufficed for this topic. In the general case though, this task can prove to be much more complex, as we discuss in section 6.2 on the non-linearly evolving topic.

\subsubsection{Messages Extraction}

This stage consists of three sub-stages. In the first we try to identify the message types that exist in the input documents, while in the second we try to fill in the messages' arguments with the instances of ontology concepts which were identified in the previous stage. The third sub-stage includes the identification of the temporal expressions that might exist in the text, and the normalization of the messages referring time, in relation to the publication time. In this topic however we did not identify any temporal expressions that would alter the messages referring time, which was set equal to the messages' publication

\footnotetext{
${ }^{19}$ http: //www . ellogon.org
} 
In the following we will assume that we have two messages of type performance:

performance $_{1}$ (of_whom 1 , in_what 1 , time__pan 1 , value 1 )

performance $_{2}$ (of_-whom 2 , in_what 2 , time_-span 2 , value 2 )

The specifications for the relations are the following:

\begin{tabular}{|c|c|c|}
\hline Relation Name: & AGREEMENT & DISAGREEMENT \\
\hline Relation Type: & Synchronic & Synchronic \\
\hline Pairs of messages: & $\{<$ performance, performance $>\}$ & $\{<$ performance, performance $>\}$ \\
\hline $\begin{array}{l}\text { Constraints on the } \\
\text { arguments: }\end{array}$ & $\begin{array}{l}\left(\text { of_whom }_{1}=\text { of }_{-} \text {whom }_{2}\right) \wedge \\
\left(\text { in_what }_{1}=\text { in_what }_{2}\right) \wedge \\
\left(\text { time_span }_{1}=\text { time_span }_{2}\right) \wedge \\
\left(\text { value }_{1}=\text { value }_{2}\right)\end{array}$ & $\begin{array}{l}\left(\text { of_whom }_{1}=\text { of }_{-} \text {whom }\right. \\
2) \wedge \\
\left(\text { in_what }_{1}=\text { in_what }_{2}\right) \wedge \\
\left(\text { time }_{-} \text {span }_{1}=\text { time_span }_{2}\right) \wedge \\
\left(\text { value }_{1} \neq \text { value }_{2}\right)\end{array}$ \\
\hline Relation Name: & POSITIVE GRADUATION & NEGATIVE GRADUATION \\
\hline Relation Type: & Diachronic & Diachronic \\
\hline Pairs of messages: & $\{<$ performance, performance $>\}$ & $\{<$ performance, performance $>\}$ \\
\hline $\begin{array}{l}\text { Constraints on the } \\
\text { arguments: }\end{array}$ & $\begin{array}{l}\left(\text { of }_{-} \text {whom }_{1}=\text { of }_{-} \text {whom }\right. \\
\text { (in_what }\end{array}$ & $\begin{array}{l}\left(\text { of_whom }_{1}=\text { of }_{-} \text {whom }\right. \\
\text { (in_what } \\
\left(\text { in }_{1}=\text { in_what }_{2}\right) \wedge \\
\left(\text { time_span }_{1}=\text { time_span }_{2}\right) \wedge \\
\left(\text { value }_{1}>\text { value }_{2}\right)\end{array}$ \\
\hline
\end{tabular}

Additionally, the messages should satisfy as well the constraints on the source and referring time in order to be candidates for a Synchronic or Diachronic Relation. In other words, the messages $m_{1}$ and $m_{2}$ will be candidates for a Synchronic Relation if

$$
\begin{aligned}
& \left|m_{1}\right|_{\text {source }}=\left|m_{2}\right|_{\text {source }} \\
& \left|m_{1}\right|_{\text {ref_time }}=\left|m_{2}\right|_{\text {ref_time }}
\end{aligned}
$$

and candidates for a Diachronic Relation if

$$
\begin{aligned}
& \left|m_{1}\right|_{\text {source }}=\left|m_{2}\right|_{\text {source }} \\
& \left|m_{1}\right|_{\text {ref_time }}>\left|m_{2}\right|_{\text {ref_time }}
\end{aligned}
$$

Figure 7: Specifications of Synchronic and Diachronic Relations for the linearly evolving topic

time. This is natural to expect, since each document is concerned only with the description of a particular football match. We can thus consider that this stage consists effectively from two sub-stages.

Concerning the first sub-stage, i.e. the identification of the message types, we approached it as a classification problem. From a study that we carried out, we concluded that in most of the cases the mapping from sentences to messages was one-to-one, i.e. in most of the cases one sentence corresponded to one message. Of course, there were cases in which one message was spanning more than one sentence, or that one sentence was containing more than one message. We managed to deal with such cases during the arguments' filling sub-stage.

In order to perform our experiments we used a bag-of-words approach according to which we represented each sentence as a vector from which the stop-words and the words with low frequencies (four or less) were removed. We performed four series of experiments. The first two series of experiments used only lexical features, namely the words of the sentences both stemmed and unstemmed. In the last two series of experiments we enhanced the vectors by adding to them semantic information as well; as semantic features we used the $N E$ types that appear in the sentence. In each of the vectors we appended the class of the sentence, i.e. the type of message; in case a sentence did not correspond to a message we labeled that vector as belonging to the class None.

In order to perform the classification experiments we used the WEKA platform (Witten and Frank 2000). The Machine Learning algorithms that we used 
were Naïve Bayes, LogitBoost and SMO. For the last two algorithms, apart from the default configuration, we performed more experiments concerning several of their arguments. For all experiments we performed a ten-fold cross-validation with the annotated corpora that we had. Ultimately, the algorithm that gave the best results was the SMO with the default configuration for the unstemmed vectors which included information on the NE types. The fact that the addition of the NE types increases the performance of the classifier, is only logical to expect since the NE types are used as arguments in the vast majority of the messages. On the other hand, the fact that by using the unstemmed words, instead of their stems, increases the performance of the classifier is counterintuitive. The reason behind this discrepancy is the fact that the SKEL stemmer (Petasis et al. 2003) that we have used, was a general-purpose one having thus a small coverage for the topic of football news.

The final sub-stage is the filling in of the messages' arguments. In order to perform this stage we employed several domain-specific heuristics. Those heuristics take into account the constraints of the messages, if such constraints exist. As we noted above, one of the drawbacks of our classification approach is that there are some cases in which we do not have an one-to-one mapping from sentences to messages. During this stage of message extraction we used heuristics to handle many of these cases.

In Table 4 we show the final performance of the messages' extraction stage as a whole, when compared against manually annotated messages on the corpora used. Those measures concern only the message types, excluding the class None messages.

$\begin{array}{lll}\text { Precision } & : & 91.12 \% \\ \text { Recall } & : & 67.79 \% \\ \text { F-Measure } & : & 77.74 \%\end{array}$

Table 4: Final evaluation of the messages' extraction stage

\subsubsection{Relations Extraction}

The final stage towards the creation of the grid is the extraction of the relations. As is evident from Figure 7, once we have identified the messages in each document and placed them in the appropriate position in the grid, then it is fairly straightforward, through their specifications, to identify the cross-document relations among the messages. In order to achieve that, we implemented a system written in Java. This system takes as input the extracted, from the previous stage, messages and it applies the algorithm, which represents the specifications of the relations, in order to extract the SDRs. Ultimately, through this system we manage to represent the grid, which carries an essential role for our summarization approach. In section 7 we analyze the fact that the creation of the grid, essentially, consists the Document Planning stage, which is the first out of three stages of a typical NLG system architecture (Reiter and Dale 2000).

Concerning the statistics of the extracted relations, these are presented in Table 5. As can be seen from that table, the evaluation results for the relations, when compared with those of the messages, are somewhat lower. This fact can be attributed to the argument extraction subsystem, which does not perform as well as the message classification subsystem. 


$\begin{array}{lll}\text { Precision } & : & 89.06 \% \\ \text { Recall } & : & 39.18 \% \\ \text { F-Measure } & : & 54.42 \%\end{array}$

Table 5: Recall, Precision and F-Measure on the relations

In this section we have examined how our methodology for the creation of summaries from evolving events, presented in section 3 , is applied to a linearly evolving topic, namely that of the descriptions of football matches. As we have said in the introduction of this section, this topic was chosen for its virtue of not being very complex. It was thus an ideal topic for a first application of our methodology. In the next section we will move forward and try to apply our methodology into a much more complex topic which evolves non-linearly.

\section{Case Study II: Non-linear Evolution}

The topic that we have chosen for our second case study is the terrorist incidents which involve hostages. The events that belong to this topic do not exhibit a periodicity concerning their evolution, which means that they evolve in a nonlinear fashion. Additionally, we wouldn't normally expect the sources to describe synchronously each event; in contrast, each source follows its own agenda on describing such events. This is best depicted in the second part of Figure 2 (page 7). In this graph we have the reports for an event which concerns a terrorist group in Iraq that kept as hostages two Italian women threatening to kill them, unless their demands were fulfilled. In the figure we depict 5 sources. The number of reports that each source is making varies from five to twelve, in a period of about 23 days.

In this section we will once again describe the topic analysis phase, i.e. the details on the collection of the corpus, the creation of the topic ontology, and the creation of the specifications for the messages and the relations. Then we will describe the system we implemented for extracting the instances of the ontology concepts, the messages and the relations, in order to form the grid.

\subsection{Topic Analysis}

The aim of the topic analysis phase, as we have thoroughly analyzed in section 3.1 and followed in the previous case-study, is to collect an initial corpus for analysis, create the ontology for the topic and create the specifications for the messages and the relations, as well as the annotation of the corpus.

\subsubsection{Corpus Collection}

The events that fall in the topic of terrorist incidents that involve hostages are numerous. In our study we decided to concentrate on five such events. Those events include the hijacking of an airplane from the Afghan Airlines in February 2000, a Greek bus hijacking from Albanians in July 1999, the kidnapping of two Italian reporters in Iraq in September 2004, the kidnapping of a Japanese group in Iraq in April 2004, and finally the hostages incident in the Moscow theater by a Chechen group in October 2004. In total we collected and examined 163 
articles from 6 sources. ${ }^{20}$ Table 6 presents the statistics, concerning the number of documents and words contained therein, for each event separately.

\begin{tabular}{|l|r|l|}
\hline Event & Documents & Words \\
\hline Airplane Hijacking & 33 & 7008 \\
Bus Hijacking & 11 & 12416 \\
Italians Kidnaping & 52 & 21200 \\
Japanese Kidnaping & 18 & 10075 \\
Moscow Theater & 49 & 21189 \\
\hline
\end{tabular}

Table 6: Number of documents, and words contained therein, for each event.

\subsubsection{Ontology Creation}

As in the previous topic examined, we created the ontology following the formal guidelines that exist in the field of ontology building, a summary of which we presented in section 3.1. The concepts of the implemented ontology are connected with is-a relations. An excerpt of the final ontology can be seen in Figure 8.

$\begin{array}{lcc}\text { Person } & \text { Place } & \text { Vehicle } \\ \text { Offender } & \text { Location of Conduct } & \text { Bus } \\ \text { Hostage } & \text { Country } & \text { Plane } \\ \text { Demonstrators } & \text { City } & \text { Car } \\ \text { Rescue Team } & \text { Armament } & \text { Media } \\ \text { Relatives } & \text { Explosive } & \text { Newspaper/Press } \\ \text { Professional } & \text { Gas } & \text { Radio } \\ \text { Governmental Executive } & \text { Gun } & \text { Internet } \\ & \text { Tank } & \text { TV }\end{array}$

Figure 8: An excerpt from the topic ontology for the non-linearly evolving topic

\subsubsection{Messages' Specifications}

After the creation of the ontology, our methodology requires that we create the messages' specifications. We would like to remind again that this process involves two main stages: providing a list with the messages types, and providing the full specifications for each message. During this process we focused, as in the previous topic, on the most important messages, i.e. the ones that we believed the final readers of the summary would be mainly interested in. The messages also had to reflect the evolution of the event. Some of the messages that we defined, had a very limited frequency in the corpora examined, thus we deemed them as unimportant, eliminating them from our messages' pool. At the end of this process we concluded on 48 messages which can be seen in Table 7. Full specifications for two particular messages can be seen in Figure 9.

\footnotetext{
${ }^{20}$ The sources we used were the online versions of news broadcasting organizations: the Greek version of the BBC (http://www.bbc.co.uk/greek/), the Hellenic Broadcasting Corporation (http://www.ert.gr), the Macedonian Press Agency (http://www.mpa.gr); a web portal (http://www.in.gr); and the online versions of two news papers: Eleftherotypia (http://www.enet.gr) and Ta Nea (http://www.tanea.gr).
} 
The first one is the negotiate message, and its semantic translation is that a person is negotiating with another person about a specific activity. The second message, free, denotes that a person is freeing another person from a specific location, which can be either the Place or the Vehicle ontology concepts. Similar specifications were provided for all the messages.

\begin{tabular}{|l|l|l|l|l|}
\hline free & ask_for & located & assure & take_on_responsibility \\
\hline kill & aim_at & inform & explode & physical_condition \\
\hline hold & kidnap & organize & be_afraid & speak_on_the_phone \\
\hline deny & arrive & announce & pay_ransom & take_control_of \\
\hline enter & arrest & transport & escape_from & give_deadline \\
\hline help & armed & negotiate & stay_parked & block_the_way \\
\hline meet & leave & threaten & interrogate & hospitalized \\
\hline start & end & work_for & give_asylum & head_towards \\
\hline put & return & hijack & encircle & prevent_from \\
\hline lead & accept & trade & & \\
\hline
\end{tabular}

Table 7: Message types for the linearly evolving topic.

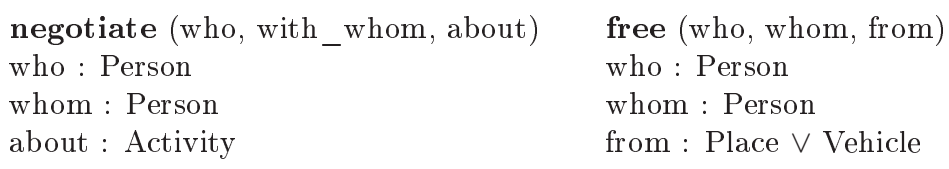

Figure 9: An example of message specifications for the non-linearly evolving topic.

\subsubsection{Relations' Specifications}

The final step during the topic analysis phase is to provide the specifications for the Synchronic and Diachronic Relations. As we have explained in section 4, Synchronic relations hold between messages that have the same referring time. In the case study examined in the previous section, we did not have any temporal expressions in the text that would alter the referring time of the messages in relation to the publication time. In this topic, we do have such expressions. Thus, Synchronic relations might hold between distant in time documents, as long as the messages' referring time is the same.

Concerning the Diachronic relations, in the previous topic we examined only relations that had temporal distance only one, i.e. we examined Diachronic relations that held only between messages found in documents, from the same source, that had been published consecutively. In this topic we have relaxed this requirement. This means that messages which have a distant referring time can be considered as candidates for a connection with a Diachronic relation. The reason for doing this is that, in contrast with the previous topic, in this topic we expect the actions of the entities to have an effect which is not "localized" in time, but can affect much later actions. This is a direct consequence of the fact that the events that belong to this topic, have a short deployment time, usually some days. In the previous topic, the events spanned several months.

Another difference is that in the previous topic we examined only relations that hold between the same message types. In this topic we also examine SDRs 
that connect messages with different message types. In the end of this process we identified 15 SDRs which can be seen in Table 8. Examples of actual relations' specifications can be seen in Figure 10.

$\begin{array}{ll}\begin{array}{l}\text { Synchronic Relations } \\ \text { (same message types) }\end{array} & \\ \text { - AGREEMENT } & \\ \text { - ELABORATION } & \text { Diachronic Relations } \\ \text { - DISAGREEMENT } & \text { (different message types) } \\ \text { - SPECIFICATION } & - \text { CAUSE } \\ & - \text { FULFILLMENT } \\ \text { Diachronic Relations } & - \text { JUSTIFICATION } \\ \text { (same message types) } & - \text { CONTRIBUTION } \\ \text { - REPETITION } & - \text { CONFIRMATION } \\ \text { - CHANGE OF PERSPECTIVE } & - \text { MOTIVATION } \\ \text { - CONTINUATION } & \\ \text { - IMPROVEMENT } & \\ \text { - DEGRADATION } & \end{array}$

Table 8: Synchronic and Diachronic Relations in the non-linearly evolving topic

Once the topic ontology, as well as the specifications of the messages and the relations had been provided, then we proceeded with the final step of the topic analysis phase of our methodology, which is annotation of the corpora, as was explained in section 3.1. We would like to add that the total amount of time required for the topic analysis phase was six months for a part-time work of two people.

\subsection{Implementation}

Having performed the topic analysis phase, the next phase involves the computational extraction of the messages and relations that will constitute the grid, forming thus the Document Planning stage, the first out of three, of a typical NLG architecture. As in the previous topic, our implementation is according to the same general architecture presented in section 3 (see also Figure 3). The details though of the implementation differ, due to the complexities that this topic exhibits. These complexities will become apparent in the rest of this section.

\subsubsection{Preprocessing}

The preprocessing stage, as in the previous case study, is a quite straightforward process. It also involves a tokenization and a sentence splitting component, but in this case study it involves as well a part-of-speech tagger. The information yielded from this stage will be used in the entities recognition and classification as well as in the messages' extraction stages, during the creation of the vectors. We would like to note again that for this stage, as well as for the next two, the ELLOGON platform (Petasis et al. 2002) was used. 
In the following we will assume that we have the following messages

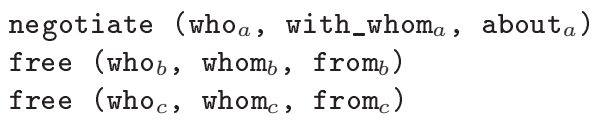

The specifications for the relations are the following:

\begin{tabular}{|c|c|c|}
\hline Relation Name: & AGREEMENT & POSITIVE EVOLUTION \\
\hline Relation Type: & Synchronic & Diachronic \\
\hline Pairs of messages: & $\{<$ free, free $>\}$ & $\{<$ negotiate, free $>\}$ \\
\hline $\begin{array}{l}\text { Constraints on the } \\
\text { arguments: }\end{array}$ & $\begin{array}{l}\left(\text { who }_{b}=\text { who }_{c}\right) \wedge \\
\left(\text { whom }_{b}=\text { whom }\right. \\
\left(\text { from }_{b}\right) \wedge \\
\left.\text { from }_{c}\right) \wedge\end{array}$ & $\begin{array}{l}\left(\text { who }_{a}=\text { who }_{b}\right) \wedge \\
\left(\text { about }_{a}=\text { free }\right)\end{array}$ \\
\hline
\end{tabular}

Additionally, the messages should satisfy as well the constraints on the source and referring time in order to be candidates for a Synchronic or Diachronic Relation. In other words, the messages $m_{1}$ and $m_{2}$ will be candidates for a Synchronic Relation if

$$
\begin{aligned}
& \left|m_{1}\right|_{\text {source }}=\left|m_{2}\right|_{\text {source }} \\
& \left|m_{1}\right|_{\text {ref_time }}=\left|m_{2}\right|_{\text {ref_time }}
\end{aligned}
$$

and candidates for a Diachronic Relation if

$$
\begin{aligned}
& \left|m_{1}\right|_{\text {source }}=\left|m_{2}\right|_{\text {source }} \\
& \left|m_{1}\right|_{\text {ref_time }}>\left|m_{2}\right|_{\text {ref_time }}
\end{aligned}
$$

Figure 10: Specifications of Synchronic and Diachronic Relations for the nonlinearly evolving topic

\subsubsection{Entities Recognition and Classification}

In the present case-study we do not have just named entities that we would like to identify in the text and categorize in their respective ontology concepts, but also general entities, which may or may not be named entities. In other words, during this stage, we are trying to identify the various textual elements in the input documents that represent an ontology concept, and classify each such textual element with the appropriate ontology concept. Take for instance the word passengers. This word, depending on the context, could be an instance of the sub-concept Hostages of the concept Persons of the ontology or it might be an instance of the sub-concept Offenders of the same ontology concept (see again Figure 8 for the ontology). It all depends on the context of the sentence that this word appears in. For example, in the sentence:

The airplane was hijacked and its 159 passengers were kept as hostages.

the word passengers ought to be classified as an instance of the Hostages ontology concept. In contrast, in the following sentence:

Three of the airplane's passengers hijacked the airplane.

the same word, passengers, ought to be classified as an instance of the $0 \mathrm{ffenders}$ ontology concept. It could be the case that under some circumstances the word passengers did not represent an instance of any ontology concept at all, for the specific topic, since this word did not participate in any instance of the messages. This is due to the fact that we have annotated only the instances of the ontology 
concepts that participate in the messages' arguments. In fact, after studying the annotated corpora, we realized that in many occasions textual elements that instantiated an ontology concept in one context did not instantiate any ontology concept in another context. Thus the task of the identification and classification of the instances of the ontology's concepts, in this case-study is much more complex than the previous one. In order to solve this problem, gazetteer lists are not enough for the present case study; more sophisticated methods ought to be used.

For this purpose we used Machine Learning based techniques. We opted in using a cascade of classifiers. More specifically, this cascade of classifiers consists of three levels. At the first level we used a binary classifier which determines whether a textual element in the input text is an instance of an ontology concept or not. At the second level, the classifier takes the instances of the ontology concepts of the previous level and classifies them under the top-level ontology concepts (such as Person or Vehicle). Finally at the third level we had a specific classifier for each top-level ontology concept, which classifies the instances in their appropriate sub-concepts; for example, in the Person ontology concept the specialized classifier classifies the instances into Offender, Hostage, etc. For all the levels of this cascade of classifiers we used the WEKA platform. More specifically we used three classifiers: Nä̈ve Bayes, LogitBoost and SMO, varying the input parameters of each classifier. We will analyze each level of the cascade separately.

After studying the annotated corpora, we saw that the textual elements that represent instances of ontology concepts could consist from one to several words. Additionally, it might also be the case that a textual element that represents an instance in one context does not represent an instance in another context. In order to identify which textual elements represent instances of ontology concepts, we created a series of experiments which took under consideration the candidate words and their context. We experimented using from one up to five tokens of the context, i.e. before and after the candidate textual elements. The information we used were token types, ${ }^{21}$ part-of-speech types, as well as their combination. After performing a tenfold cross-validation using the annotated corpora, we found that the classifier which yielded the best results was LogitBoost with 150 boost iterations, using only the token types and a context window of four tokens.

The next level in the cascade of classifiers is the one that takes as input the instances of ontology concepts found from the binary classifier, and determines their top-level ontology concept (e.g. Person, Place, Vehicle). The features that this classifier used for its vectors, during the training phase, were the context of the words, as well as the words themselves. More specifically we created a series of experiments which took into consideration one to up to five tokens before and after the textual elements, as well as the tokens which comprised the textual element. The features that we used were the token types, the part-of-speech types, and their combination. The classifier that yielded the best results, after performing a tenfold cross-validation, was LogitBoost with 100 boost iterations with a context of size one, and using as features the token types and part-of-speech types for each token.

${ }^{21}$ The types of the tokens denote whether a particular token was an uppercase or lowercase word, a number, a date, a punctuation mark, etc. 
The final level of the cascade of classifiers consists of a specialized classifier for each top-level ontology concept, which determines the sub-concepts in which the instances, classified at the previous level, belong. In this series of experiments we took as input only the nouns that were contained in each textual element, discarding all the other tokens. The combined results from the cascade of classifiers, after performing a tenfold cross-validation, are shown in Table 9. The last column in that table, represents the classifier used in the third level of the cascade. The parameter $I$ in the LogitBoost classifier represents the boost cycles. For conciseness we present only the evaluation results for each top-level ontology concept. The fact that the Person, Place and Activity concepts scored better, in comparison to the Media and Vehicle concepts, can be attributed to the fact that we did not have many instances for the last two categories to train the classifier.

\begin{tabular}{|r|l|l|l|l|}
\hline Class & Precision & Recall & F-Measure & Classifier \\
\hline Person & $75.63 \%$ & $83.41 \%$ & $79.33 \%$ & SMO \\
Place & $64.45 \%$ & $73.03 \%$ & $68.48 \%$ & LogitBoost $(\mathrm{I}=700)$ \\
Activity & $76.86 \%$ & $71.80 \%$ & $74.25 \%$ & LogitBoost $(\mathrm{I}=150)$ \\
Vehicle & $55.00 \%$ & $45.69 \%$ & $49.92 \%$ & Naïve Bayes \\
Media & $63.71 \%$ & $43.66 \%$ & $51.82 \%$ & LogitBoost $(\mathrm{I}=150)$ \\
\hline
\end{tabular}

Table 9: The combined results of the cascade of classifiers

Finally, we would like to note that apart from the above five concepts, the ontology contained three more concepts, which had a very few instances, making it inappropriate to include those concepts into our Machine Learning experiments. The reason for this is that if we included those concepts in our Machine Learning experiments we would have the phenomenon of skewed class distributions. Instead we opted in using heuristics for those categories, during which we examined the context of several candidate words. The results are shown in Table 10.

\begin{tabular}{|l|l|l|l|}
\hline Ontology Concept & Precision & Recall & F-Measure \\
\hline Public Institution & $88.11 \%$ & $91.75 \%$ & $89.89 \%$ \\
Physical Condition & $94.73 \%$ & $92.30 \%$ & $93.50 \%$ \\
Armament & $98.11 \%$ & $100 \%$ & $99.04 \%$ \\
\hline
\end{tabular}

Table 10: Evaluation for the last three ontology concepts

\subsubsection{Messages Extraction}

This stage consists of three sub-stages. At the first one we try to identify the message types that exist in the input documents, while at the second we try to fill in the messages' arguments with the instances of the ontology concepts identified in the previous stage. The third sub-stage includes the identification of the temporal expressions that might exist in the text, and the normalization of the messages' referring time, in relation to the document's publication time.

Concerning the first sub-stage, after studying the corpora we realized that we had an one-to-one mapping from sentences to message types, exactly as happened in the previous case-study. We used again Machine Learning techniques 
to classify sentences to message types. We commenced our experiments by a bag-of-words approach using, as in the previous case-study, the combination of lexical and semantic features. As lexical features we used the words of the sentences, both stemmed and unstemmed; as semantic features we used the number of the instances of each sub-concept that were found inside a sentence. This resulted in a series of four experiments, in each of which we applied the Naïve Bayes, LogitBoost and SMO algorithms of the WEKA platform. Unfortunately, the results were not as satisfactory as in the previous case study. The algorithm that gave the best results was the SMO using both the semantic and lexical features (as lexical features it used the unstemmed words of the sentences). The percentage of the message types that this algorithm managed to correctly classify were $50.01 \%$, after performing a ten fold cross validation on the input vectors. This prompted us to follow a different route for the message type classification experiments.

The vectors that we created, in this new set of Machine Learning experiments, incorporated again both lexical and semantic features. As lexical features we now used only a fixed number of verbs and nouns occurring in the sentences. Concerning the semantic features, we used two kinds of information. The first one was a numerical value representing the number of the top-level ontology concepts (Person, Place, etc) that were found in the sentences. Thus the created vectors had eight numerical slots, each one representing one of the top-level ontology concepts. Concerning the second semantic feature, we used what we have called trigger words, which are several lists of words, each one "triggering" a particular message type. Thus, we allocated six slots - the maximum number of trigger words found in a sentence - each one of which represented the message type that was triggered, if any. In order to perform our experiments, we used the WEKA platform. The algorithms that we used were again the Naïve Bayes, LogitBoost and SMO, varying their parameters during the series of experiments that we performed. The best results were achieved with the LogitBoost algorithm, using 400 boost cycles. More specifically the number of correctly classified message types were $78.22 \%$, after performing a ten-fold cross-validation on the input vectors.

The second sub-stage is the filling in of the messages' arguments. In order to perform this stage we employed several domain-specific heuristics which take into account the results from the previous stages. It is important to note here that although we have an one-to-one mapping from sentences to message types, it does not necessarily mean that the arguments (i.e. the extracted instances of ontology concepts) of the messages will also be in the same sentence. There may be cases where the arguments are found in neighboring sentences. For that reason, our heuristics use a window of two sentences, before and after the one under consideration, in which to search for the arguments of the messages, if they are not found in the original one. The total evaluation results from the combination of the two sub-stages of the messages extraction stage are shown in Table 11. As in the previous cases, we also used a tenfold cross-validation process for the evaluation of the Machine Learning algorithms.

At this point we would like to discuss the results a little bit. Although in the first sub-stage, the classification of the sentences into message types, we had $78.22 \%$ of the sentences correctly classified, the results of Table 11 diverge from that number. As we have noted earlier, the results of Table 11 contain the combined results from the two sub-stages, i.e. the classification of the sentences 


$\begin{array}{lll}\text { Precision } & : & 42.96 \% \\ \text { Recall } & : & 35.91 \% \\ \text { F-Measure } & : & 39.12 \%\end{array}$

Table 11: Evaluation for the messages extraction stage of the non-linearly evolving topic.

into message types as well as the filling in of the messages' arguments. The main reason for the divergence then, seems to be the fact that the heuristics used in the second sub-stage did not perform quite as well as expected. Additionally, we would like to note that a known problem in the area of Information Extraction (IE) is the fact that although the various modules of an IE system might perform quite well when used in isolation, their combination in most of the cases yields worst results from the expected ones. This is a general problem in the area of Information Extraction, which needs to be dealt with (Grishman 2005).

The last of the three sub-stages, in the messages extraction stage, is the identification of the temporal expressions found in the sentences which contain the messages and alter their referring time, as well as the normalization of those temporal expressions in relation to the publication time of the document which contains the messages. For this sub-stage we adopted a module which was developed earlier (Stamatiou 2005). As was mentioned earlier in this paper, the normalized temporal expressions alter the referring time of the messages, an information which we use during the extraction of the Synchronic and Diachronic Relations.

\subsubsection{Relations Extraction}

The final processing stage in our architecture is the extraction of the Synchronic and Diachronic Relations. As in the previous case-study, the implementation of this stage is quite straightforward. All that is needed to be done is the translation of the relations' specifications into an appropriate algorithm which, once applied to the extracted messages, will provide the relations that connect the messages, effectively thus creating the grid.

We implemented this stage in Java, creating a platform that takes as input the extracted messages, including their arguments and the publication and referring time. The result of this platform is the extraction of the relations. Those results are shown on Table 12. As we can see, although the F-Measures of the messages extraction stage and the relations extraction stage are fairly similar, their respective precision and recall values diverge. This is mostly caused due to the fact that small changes in the arguments of the messages can yield different relations, decreasing the precision value. The extracted relations, along with the messages that those relations connect, compose the grid. In section 7 we will thoroughly present the relation of the grid with the typical stages of an NLG component. In fact, we will show how the creation of the grid essentially constitutes the Document Planning phase, which is the first out of three of a typical NLG architecture (Reiter and Dale 2000). Additionally, in that section we will provide an example of the transformation of a grid into a textual summary. 


$\begin{array}{lll}\text { Precision } & : & 30.66 \% \\ \text { Recall } & : & 49.12 \% \\ \text { F-Measure } & : & 37.76 \%\end{array}$

Table 12: Evaluation for the relations extraction stage of the non-linearly evolving topic.

\section{Generating Natural Language Summaries from the Grid}

In section 3 we have given an overview of our methodology concerning the automatic creation of summaries from evolving events. The results of its application in two case studies have been presented in sections 5 and 6 . The core of the methodology addresses the issue of extracting the messages and the Synchronic and Diachronic Relations from the input documents, creating thus a structure we called grid. Throughout this paper we have emphasized the fact that this structure will be passed over to a generator for the creation of the final document, i.e. summary. In this section we would like to show more concretely the connection between the grid, i.e. a set messages and some SDRs connecting them, with research in Natural Language Generation. More specifically, we would like to show how a grid might be the first part of the typical three components of a generator.

According to Reiter and Dale (2000) the architecture of a Natural Language Generation system is divided into the following three stages. ${ }^{22}$

1. Document Planning. This stage is divided into two components:

(a) Content Determination. The core of this stage is the determination of what information should be included in the generated text. Essentially, this process involves the choice or creation of a set of messages (Reiter and Dale 1997, 2000) from the underlying sources.

(b) Content Structuring. The goal of this stage is the ordering of the messages created during the previous step, taking into account the communicative goals the to-be generated text is supposed to meet. To this end messages are connected with discourse relations. These latter are generally gleaned from Rhetorical Structure Theory.

2. Micro-Planning. This element is composed of the following three components:

(a) Lexicalization. This component involves the selection of the words to be used for the expression of the messages and relations.

(b) Aggregation. At this stage a decision is made concerning the level and location where a message is supposed to be included: in a same paragraph, a sentence or at the clause level. Furthermore, unnecessary or redundant information is factored out, eliminating thus repetition and making the generated text run more smoothly. This component takes also the relations holding between the messages into account.

\footnotetext{
${ }^{22}$ Rather than following Reiter and Dale's (1997) original terminology we will follow the terms they used in Reiter and Dale (2000), as they seem to be more widely accepted.
} 
(c) Referring Expressions Generation. The goal of this stage is to determine the information to be given (noun vs. pronoun) in order to allow the reader to discriminate a given object (the referent) from a set of alternatives (a "cat" vs "the cat" vs "it").

3. Surface Generation. In this, final, stage the actual paragraphs and sentences are created according to the specifications of the previous stage. This is in fact the module where the knowledge about the grammar of the target natural language is encoded.

Having provided a brief summary of the stages involved in a typical generator, we would like now to proceed to show how the creation of the grid might indeed, together with the communicative goal, become the starting point, i.e. the document planning, of an NLG system.

According to Reiter and Dale (1997), the main task of content determination resides in the choice of "the entities, concepts and relations" from the "underlying data-sources". Once this is done, we need to structure them.

Having established the entities, concepts, and relations we need make use of, we can then define a set of messages which impose structure over these elements. (Reiter and Dale 2000, p 61)

The reader should be aware that the "relations" mentioned here are different in nature from the rhetorical relations to be established during the content structuring stage. This being said, let us now try to translate the above given concepts with the ones of our own research. The "underlying data-sources", in our case, are the input documents from the various sources, describing the evolution of an event, which we want to summarize. The "entities and concepts" in our case are defined in terms of the topic ontology, to be used later on as arguments of the messages. Reiter and Dale's (2000) "relations" correspond to our message types. The structuring is identical in both cases. Hence we can conclude that the two structures are essentially identical in nature. The creation of the messages, which concludes the content determination sub-stage, is performed in our case during the messages extraction step of the implementation phase.

The goal of content structuring, the next stage, is to impose some order on the messages selected during the content determination sub-stage, by taking communicative goals into account. This is usually achieved by connecting the messages via so called "discourse relations". It should be noted however that:

There is no consensus in the research literature on what specific discourse relations should be used in an NLG system. (Reiter and Dale 1997, p 74)

Nevertheless, according to Reiter and Dale probably the most common set of relations used to establish coherence and achieve rhetorical goals, is the one suggested by the Rhetorical Structure Theory of Mann and Thompson (1987, 1988), to which they add that

[ . . . many developers modify this set to cater for idiosyncrasies of their particular domain and genre. 
The above are, in fact, fully in line with our decision to connect the created messages not with any conventional, a priori set of discourse relations, but rather with what we have called Synchronic and Diachronic Relations ${ }^{23}$ the latter providing in essence an ordering of the messages scattered throughout the various input documents. Hence we can say, that this component fulfils the same function as the content structuring component of the document planning of the Reiter and Dale model, as it connects messages with SDRs.

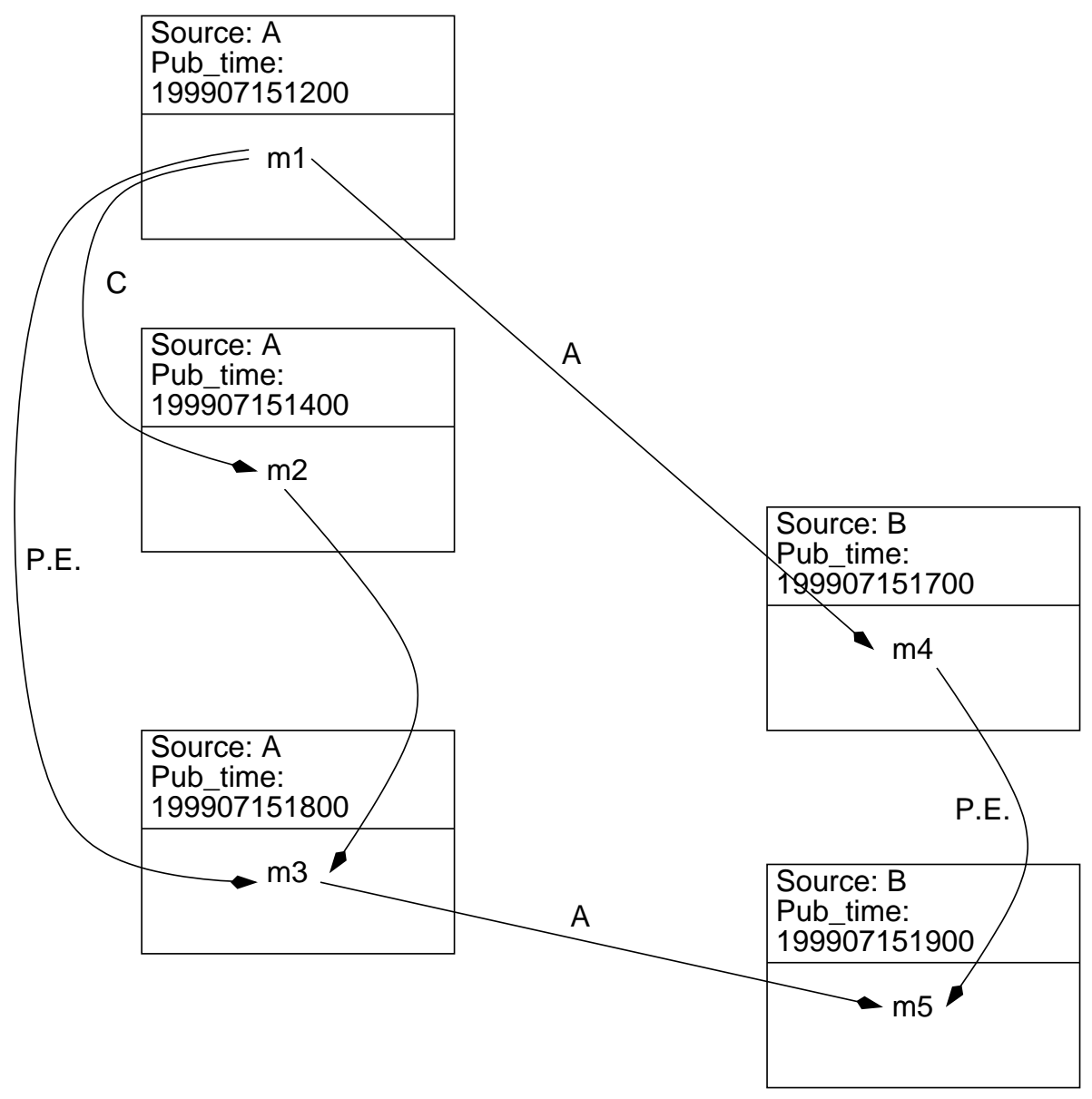

Figure 11: A tiny excerpt of the created grid for the non-linearly evolving topic. The messages $\mathrm{m} 1-\mathrm{m} 5$ correspond to sentences $\mathrm{s}_{1}-\mathrm{s}_{5}$ of Table 13. A: Agreement, C: Continuation, P.E.: Positive Evolution.

From the previous analysis we have concluded that the creation of the grid, i.e. the identification of messages and their connection with SDRs, constitutes in essence the first stage of a Natural Language Generation system. Now we would like to show how such a grid can be transformed into a text summary.

\footnotetext{
${ }^{23}$ While the SDRs are by no means a modified set of RST relations, they were certainly inspired by them. In section 8 we will see their respective similarities and differences, as well as where precisely SDRs provide some improvements over RST relations.
} 


\begin{tabular}{|c|c|}
\hline & Sentence \\
\hline $\mathrm{s}_{1}$ & $\begin{array}{l}\text { According to officials, the negotiations between the hijackers and the } \\
\text { negotiating team have started, and they focus on letting free the chil- } \\
\text { dren from the bus. }\end{array}$ \\
\hline $\mathrm{s}_{2}$ & $\begin{array}{l}\text { At the time of writing, the negotiations between the hijackers and the } \\
\text { negotiating team, for the freeing of the children from the bus, continue. }\end{array}$ \\
\hline $\mathrm{s}_{3}$ & $\begin{array}{l}\text { The negotiating team managed to convince the hijackers to let free the } \\
\text { children from the bus. }\end{array}$ \\
\hline $\mathrm{s}_{4}$ & $\begin{array}{l}\text { The negotiating team arrived at 12:00 and negotiates with the hijackers } \\
\text { for the freeing of the children from the bus. }\end{array}$ \\
\hline \multirow[t]{2}{*}{$\mathrm{s}_{5}$} & An hour ago the children were freed from the bus by the hijackers. \\
\hline & Message \\
\hline $\mathrm{m} 1$ & $\begin{array}{l}\text { negotiate ("negotiating team", "hijackers", "free") } \\
|\mathrm{m} 1|_{\text {source }}=\mathrm{A} ;|\mathrm{m} 1|_{\text {pub_time }}=199907151200 ; \\
|\mathrm{m} 1|_{\text {ref_time }}=199907151200\end{array}$ \\
\hline $\mathrm{m} 2$ & $\begin{array}{l}\text { negotiate ("negotiating team", "hijackers", "free") } \\
|\mathrm{m} 2|_{\text {source }}=\mathrm{A} ;|\mathrm{m} 2|_{\text {pub_time }}=199907151400 \\
|\mathrm{~m} 2|_{\text {ref_time }}=199907151400\end{array}$ \\
\hline m3 & $\begin{array}{l}\text { free ("hijackers", "children", "bus") } \\
|\mathrm{m} 1|_{\text {source }}=\mathrm{A} ;|\mathrm{m} 1|_{\text {pub_time }}=199907151800 \\
|\mathrm{~m} 1|_{\text {ref_time }}=199907151800\end{array}$ \\
\hline $\mathrm{m} 4$ & $\begin{array}{l}\text { negotiate ("negotiating team", "hijackers", "free") } \\
|\mathrm{m} 1|_{\text {source }}=\mathrm{A} ;|\mathrm{m} 1|_{\text {pub_time }}=199907151700 \\
|\mathrm{~m} 1|_{\text {ref_time }}=199907151200\end{array}$ \\
\hline $\mathrm{m} 5$ & $\begin{array}{l}\text { free ("hijackers", "children", "bus") } \\
|\mathrm{m} 1|_{\text {source }}=\mathrm{A} ;|\mathrm{m} 1|_{\text {pub_time }}=199907151900 ; \\
|\mathrm{m} 1|_{\text {ref_time }}=199907151800\end{array}$ \\
\hline
\end{tabular}

Table 13: The corresponding sentences and message instances of the $\mathrm{m} 1-\mathrm{m} 5$ of Figure 11.

In Figure 11 we provide an excerpt from the automatically built grid, of a bus hijacking event, which was thoroughly examined in section 6 .

Each rectangle represents a document annotated with information concerning the source and time of publication of the document. In this small excerpt of the grid we depict one message per source. The messages correspond to the sentences of Table 13. They are connected with the Synchronic and Diachronic relations as shown in Figure 11. Note that in order to establish a Synchronic relation between two messages reference time is taken into account rather than the time of publication of the messages. The messages for which we have a different reference time, as opposed to their publication time, are $\mathrm{m} 4$ and $\mathrm{m} 5$. This is marked explicitly by the temporal expressions "at 12:00" and "an hour ago" in sentence $\mathrm{s}_{4}$ and $\mathrm{s}_{5}$. Thus the messages $\mathrm{m} 4, \mathrm{~m} 5$ and $\mathrm{m} 1, \mathrm{~m} 3$ are connected respectively via the Synchronic relation Agreement as: (1) they belong to different sources, (2) they have the same reference time, and (3) their arguments fulfil the constraints presented in Figure 10. A similar syllogism applies for the Diachronic relations. Hence, the messages $\mathrm{m} 1$ and $\mathrm{m} 3$ are connected via a Positive Evolution Diachronic relation because: (1) they belong to the same source, (2) they have different reference times, and (3) their arguments fulfil 
the constraints presented in Figure 10. Once such a grid is passed to the NLG component, it may lead to the following output, i.e. summary.

According to all sources, the negotiations between the hijackers and the negotiating team, for the freeing of the children, started at 12:00. The continuous negotiations resulted in a positive outcome at 18:00 when the hijackers let free the children.

\section{Related Work}

In this paper we have presented a methodology which aims at the automatic creation of summaries from evolving events, i.e. events which evolve over time and which are being described by more than one source. Of course, we are not the first ones to incorporate directly, or indirectly, the notion of time in our approach of summarization. For example, Lehnert (1981), attempts to provide a theory for what she calls narrative summarization. Her approach is based on the notion of "plot units", which connect mental states with various relations, likely to be combined into highly complex patterns. This approach applies for single documents. Bear in mind though that the author does not provide any implementation of her theory. More recently, Mani (2004) attempts to revive this theory, although, again we lack a concrete implementation validating the approach.

From a different viewpoint, Allan et al. (2001) attempt what they call temporal summarization. In order to achieve this goal, they start from the results of a Topic Detection and Tracking system for an event, and order sentences chronologically, regardless of their origin, creating thus a stream of sentences. Then they apply two statistical measures, usefulness and novelty, to each ordered sentence. The aim being the extraction of sentences whose score is above a given threshold. Unfortunately, the authors do not take into account the document sources, and they do not consider the evolution of the events; instead they try to capture novel information. Actually, what Allan et al. (2001) do is to create an extractive summary, whereas we aim at the creation of abstractive summaries. $^{24}$

As mentioned already, our work requires some domain knowledge, acquired during the so called "topic analysis" phase, which is expressed conjointly via the ontology, and the specification of messages and relations. One such system based on domain knowledge is Summons (Radev and McKeown 1998; Radev 1999). The main domain specific knowledge of this system comes from the specifications of the MUC conferences. SUMMONs takes as input several MUC templates and, having applied a series of operators, it tries to create a baseline summary, which is then enhanced by various named entity descriptions collected from the Internet. Of course, one could argue that the operators used by SUMMONS resemble our SDRs. However, this resemblance is only superficial, as our relations are divided into Synchronic and Diachronic ones, thus reporting similarities and differences in two opposing directions.

Concerning the use of relations, there have been several attempts in the past to try to incorporate them, in one form or another, in summary creation. Salton

\footnotetext{
${ }^{24}$ Concerning the difference between these two kind of summaries see Afantenos et al. (2005a, p 160).
} 
et al. (1997), for example, try to extract paragraphs from a single document by representing them as vectors and assigning a relation between the vectors if their similarity exceeds a certain threshold. They present then various heuristics for the extraction of the best paragraphs.

Finally, Radev (2000) proposed the Cross-document Structure Theory (CST) taking into account 24 domain independent relations existing between various text units across documents. In a later paper Zhang et al. (2002) reduce the set to 17 relations and perform some experiments with human judges. These experiments produce various interesting results. For example, human judges annotate only sentences, ignoring completely any other textual unit (phrases, paragraphs, documents) suggested by the theory. Also, the agreement between judges concerning the type of relation holding between two connected sentences is rather small. Nevertheless, Zhang et al. (2003) and Zhang and Radev (2004) continued to explore these issues by using Machine Learning algorithms to identify crossdocument relations. They used the Boosting algorithm and the F-measure for evaluation. The results for six classes of relation, vary from $5.13 \%$ to $43.24 \%$. However, they do not provide any results for the other 11 relations. ${ }^{25}$ Concerning the relations we should note, that, while a general pool of cross-document relations might exist, we believe that, in contrast to Radev (2000), they are domain dependent, as one can choose from this pool the appropriate subset of relations for the domain under consideration, possibly enhancing them with completely domain specific relations to suit one's own needs. Another significant difference from our work, is that we try to create summaries that show not only the evolution of an event, but also the similarities or differences of the sources during the event's evolution.

Another kind of related work that we would like to discuss here is the Rhetorical Structure Theory (RST). Although RST has not been developed with automatic text summarization in mind, it has been used by Marcu $(1997,2000)$ for the creation of extractive single-document summaries. In this section we will not discuss Marcu's work since it concerns the creation of summaries from single documents. ${ }^{26}$ Instead, in the following we will attempt a comparison of our approach with RST, specifying their respective similarities and differences, as well as the points where our approach presents an innovation with regard to RST. We would like though to issue a warning to the reader that, even if we claim that our approach extends the Rhetorical Structure Theory, we are fully aware of our intellectual debts towards the authors of RST. The innovations we are claiming here are somehow linked to the specific context of summarizing evolving events. In fact, the decisions we have made have recently found a kind of assent by one of the creators of RST in a paper entitled "Rhetorical Structure Theory: Looking Back and Moving Ahead" (Taboada and Mann 2006). What we mean by this is that what the authors provide as innovations to be considered in the future of RST, have, in a sense, been implemented by us, be it though in the context of the text summarization of evolving events. This being said, let us proceed with a brief description of RST and the similarities, differences and

\footnotetext{
${ }^{25} \mathrm{By}$ contrast, in our work the F-Measure for all the relations, is $54.42 \%$ and $37.76 \%$ respectively for the topics of the football matches and the terrorist incidents involving hostages.

${ }^{26}$ The interested reader should take a look at his works (e.g. Marcu 1997, 2000, 2001). For a comparison of this and other related works you may consider taking a look at Mani (2001) or Afantenos et al. (2005a).
} 
innovations of our work.

Rhetorical Structure Theory has been introduced by Mann and Thompson $(1987,1988)$. It was originally developed to address the issue of text planning, or text structuring in NLG, as well as to provide a more general theory of how coherence in texts is achieved (Taboada and Mann 2006). This theory made use of a certain number of relations, which carried semantic information. Examples of such relations are Contrast, Concession, Condition, etc. The initially proposed set contained 24 relations (Mann and Thompson 1988); today we have 30 relations (Taboada and Mann 2006). Each relation holds between two or more segments, units of analysis, generally clauses. The units, schemata, are divided into nuclei and satellites, depending on their relative importance. Only the most prominent part, the nucleus, is obligatory. Relations can hold not only between nuclei or satellites but also between any of them and an entire schema (a unit composed of a nucleus and a satellite), hence, potentially we have a tree.

As mentioned already, RST was developed, with the goal of Natural Language Generation: "It was intended for a particular kind of use, to guide computational text generation" (Taboada and Mann 2006, p 425). In fact, this is also what we had in mind, when we developed our approach. As explained in section 3.1.2, our notion of messages was inspired by the very same notion used in the domain of NLG. In addition, our messages are connected with Synchronic and Diachronic Relations, forming thus what we have called a grid, that is a structure to be handed over to the surface generation component of an NLG system in order to create the final summary.

The point just made is one of similarity between the two approaches. Let us now take a look at a point where we believe to be innovative. As mentioned already, RST relations hold generally between clauses. ${ }^{27}$ As Taboada and Mann (2006) write, choosing the clause as the unit of analysis works well in many occasions; but they concede that occasionally, this has some drawbacks. Actually they write ( $\mathrm{p} 430)$ that:

We do not believe that one unit division method will be right for everyone; we encourage innovation.

This is precisely the point where we are innovative. Our units of analysis are not clauses, or any other textual element, rather we have opted for messages as the units of analysis, which, as mentioned in section 3.1.2, impose a structure over the entities found in the input text.

While the units of analysis in RST are divided into nuclei and satellites, our units of analysis - messages - do not have such a division. This is indeed a point where RST and our approach differ radically. In RST nuclei are supposed to represent more prominent information, compared to satellites. In our own approach this is remedied through the use of a query (see section 3) from the user. What we mean by this is that we do not a priory label the units of analysis in terms of relative importance, instead we let the user do this. In a sense, we determine prominence via a query, which is then thoroughly analyzed by our system, so that it will then be mapped to the messages, and accordingly to the SDRs that connect them, and describe best the query.

Let us now say a few words concerning the taxonomy of relations. As explained in section 4 we divide our relations into Synchronic and Diachronic

\footnotetext{
${ }^{27}$ And, of course, between spans of units of analysis.
} 
relations. In addition we assume that these relations are domain-dependant, in the sense that we have to define SDRs for each new topic. While a stable set of topic independent SDRs might exist, we do not make such a claim. By contrast, RST relations are domain independent. The initial set of RST relations had a cardinality of 24, with 6 more relations added more recently, which leaves us with 30 RST relations. This set is considered by many researchers, though not by all, as a fixed set. Yet, this is not what was intended by the developers of Rhetorical Structure Theory. As pointed out by Mann and Thompson (1988, p 256): "no single taxonomy seems suitable", which encourages our decision to have topic sensitive SDRs, that is, SDRs being defined for each new topic, in order to fulfil the needs of each topic. In fact, Taboada and Mann (2006, p 438) claim that:

There may never be a single all-purpose hierarchy of defined relations, agreed upon by all. But creating hierarchies that support particular technical purposes seems to be an effective research strategy.

which somehow supports our decisions to introduce topic sensitive SDRs.

Another point where RST seems to hold similar views as we do, is the semantics of the relations. In both cases, relations are supposed to carry semantic information. In our approach this information will be exploited later on by the generator for the creation of the final summary, whereas in RST it is supposed to show the coherence of the underlying text and to present the authors' intentions, facilitating thus the automatic generation of text. While the relations carry semantic information in both cases, in RST they were meant above all to capture the authors' intentions. We do not make such a claim.

A final, probably minor point in which the two approaches differ is the resulting graph. In RST the relations form a tree, whilst in our theory the relations form a directed acyclic graph. This graph, whose messages are the vertices and the relations the edges, forms basically what we have called the grid, that is the structure to be handed down to the NLG component.

\section{Conclusions and Future Work}

In this paper we have presented a novel approach concerning the summarization of multiple documents dealing with evolving events. One point we focused particularly on was the automatic detection of the Synchronic and Diachronic Relations. As far as we know, this problem has never been studied before. The closest attempt we are aware of is Allan et al.'s (2001) work, who create what they call temporal summaries. Nevertheless, as explained in section 8 , this work does not take into account the event's evolution. Additionally, they are in essence agnostic in relation to the source of the documents, since they concatenate all the documents, irrespective of source, into one big document in which they apply their statistical measures.

In order to tackle the problem of summarizing evolving events, we have introduced the notions of messages and Synchronic and Diachronic Relations (SDRs). Messages impose a structure over the instances of the ontology concepts found in the input texts. They are the units of analysis for which the SDRs hold. Synchronic relations hold between messages from different sources with identical reference time, whilst Diachronic relations hold between messages from the same 
source with different reference times. In section 2 we provided definitions for the notions of topic, event and activities, borrowing from the terminology of Topic Detection and Tracking research. We also drew a distinction concerning the evolution of the events, dividing them into linear and non-linear events. In addition, we made a distinction concerning the report emission rate of the various sources, dividing them into synchronous and asynchronous emissions. We also provided a formal framework to account for the notions of linearity and synchronicity. Finally, we have shown how these distinctions affect the identification of the Synchronic and Diachronic Relations.

In section 3 we have presented our methodology behind the implementation of a system that extracts Synchronic and Diachronic Relations from descriptions of evolving events. This methodology is composed of two phases: the topic analysis phase and the implementation phase, presented in the subsections 3.1 and 3.2 respectively. In sections 5 and 6 we described two case-studies for a linearly and non-linearly evolving topic, which implement the proposed methodology. While the results are promising in both cases, there is certainly room for improvement for certain components. The tools incorporated for the implementation include the WEKA platform for the training of the Machine Learning algorithms, as well as the ELLOGON platform used for the annotation stage of the topic analysis phase and the development of the module used in the extraction of the messages.

In section 7 we have shown how the creation of the grid, i.e. the extraction of the messages and their connection via Synchronic and Diachronic Relations, forms essentially the Document Planning stage, i.e. the first out of the three stages of a typical NLG system (Reiter and Dale 2000). Finally, in section 8 we have presented related works, emphasizing the relationship between Rhetorical Structure Theory and our approach. We have shown the respective similarities and differences between the two, highlighting the innovative aspects of our approach. These innovations are in line with what one of the creators of RST presents as one the points that ought to be considered for the future of RST in a recent paper entitled "Rhetorical Structure Theory: Looking Back and Moving Ahead" (Taboada and Mann 2006). Again, we would like though to emphasize that, while certain parts of our approach have been inspired by RST, the approach as a whole should not be considered as an attempt of improvement of RST. In a similar vein, our innovations, should not be considered as an extension of RST. Instead it should merely be viewed as a new kind of methodology to tackle the problem of summarization of evolving events, via Synchronic and Diachronic Relations.

As mentioned in section 3 we have presented a general architecture of a system which implements the proposed approach. The implementation of the NLG subsystem has not been completed yet. The Micro-Planning and Surface Generation stages are still under development. The completion of the NLG component is an essential aspect of our current work. Even if the results of the entities-, message-, and relation-extraction components - which are part of the summarization core - yield quite satisfactory results, we need to qualitatively evaluate our summaries. Yet, this will only be possible once the final textual summaries are created, and this requires the completion of the NLG component.

As shown in the evaluation of the system's components, the results concerning the summarization core are quite promising. Obviously, there is still 
room for improvement. The component that seems to need most urgent consideration is the arguments filling component. Up to now we are using heuristics which take into account the sentences' message types, returned by the dedicated classifier, as well as the extracted entities, resulting from the various classifiers used (see section 6.2.2). This method does seem to be brittle, hence additional methods might be needed to tackle this problem. One idea would be to study various Machine Learning methods taking into account previously annotated messages, i.e. message types and their arguments. Another module needing improvement is the entity-extraction component, especially the first classifier (the binary classifier) of the cascade of classifiers presented.

Concerning the summarization core, as we have shown in the evaluation of the several components included in this system, the results are promising. Yet, there is still room for improvement. The component that seems to need an immediate consideration is the arguments filling one. Up till now we are using heuristics which take into consideration the message type of the sentence, as returned by the dedicated classifier, as well as the extracted entities, which are in turn the result of the various classifiers used (see section 6.2.2). This method does not seem to perform perfectly, which means that additional methods should be considered in order to tackle that problem. An idea would be the investigation of various Machine Learning methods which would take into account previously annotated messages, i.e. message types with their arguments. An additional module that needs improvement is the entities extraction component, especially the first classifier (the binary classifier) in the cascade of classifiers that we have presented.

An additional point that we would like to make concerns the nature of messages and the reduction of the human labor involved in the provision of their specifications. As it happens, the message types that we have provided for the two case studies, rely heavily on either verbs or verbalized nouns. This implies that message types could be defined automatically based mostly on statistics on verbs and verbalized nouns. Concerning their arguments, we could take into account the types of the entities that exist in their near vicinities. This is an issue that we are currently working on. Another promising path for future research might be the inclusion of the notion of messages, and possibly the notion of Synchronic and Diachronic Relations, into the topic ontology.

\section{Acknowledgments}

The first author was partially supported by a research scholarship from the Institute of Informatics and Telecommunications of NCSR "Demokritos", Athens, Greece. The authors would like to thank Michael Zock for his invaluable and copious comments on a draft of the paper. Additionally, the authors would like to thank Eleni Kapelou and Irene Doura for their collaboration on the specifications of the messages and relations for the linearly evolving topic, and Konstantina Liontou and Maria Salapata for the collaboration on the specifications of the messages and relations for the non-linearly evolving topic, as well as the annotation they have performed. Finally the authors would like to thank George Stamatiou for the implementation of the temporal expressions module on the non-linearly evolving topic. 


\section{References}

Afantenos, Stergos D., Irene Doura, Eleni Kapellou, and Vangelis Karkaletsis. 2004, May. "Exploiting Cross-Document Relations for Multi-Document Evolving Summarization." Edited by G. A. Vouros and T. Panayiotopoulos, Methods and Applications of Artificial Intelligence: Third Hellenic Conference on AI, SETN 2004, Volume 3025 of Lecture Notes in Computer Science. Samos, Greece: Springer-Verlag Heidelberg, 410-419.

Afantenos, Stergos D., Vangelis Karkaletsis, and Panagiotis Stamatopoulos. 2005a. "Summarization from Medical Documents: A Survey." Journal of Artificial Intelligence in Medicine 33 (2): 157-177 (February).

. 2005b, September. "Summarizing Reports on Evolving Events; Part I: Linear Evolution." Edited by Galia Angelova, Kalina Bontcheva, Ruslan Mitkov, Nicolas Nicolov, and Nikolai Nikolov, Recent Advances in Natural Language Processing (RANLP 2005). Borovets, Bulgaria: INCOMA, 1824.

Afantenos, Stergos D., Konstantina Liontou, Maria Salapata, and Vangelis Karkaletsis. 2005c, May. "An Introduction to the Summarization of Evolving Events: Linear and Non-linear Evolution." Edited by Bernadette Sharp, Proceedings of the 2nd International Workshop on Natural Language Understanding and Cognitive Science, NLUCS 2005. Maiami, Florida, USA: INSTICC Press, 91-99.

Allan, James, Jaime Carbonell, George Doddington, Jonathan Yamron, and Yiming Yang. 1998a, February. "Topic Detection and Tracking Pilot Study: Final Report." Proceedings of the DARPA Broadcast News Transcription and Understanding Workshop. 194-218.

Allan, James, Rahuk Gupta, and Vikas Khandelwal. 2001. "Temporal Summaries of News Stories." Proceedings of the ACM SIGIR 2001 Conference. $10-18$.

Allan, James, Ron Papka, and Victor Lavrenko. 1998b, August. "On-line New Event Detection and Tracking." Proceedings of the $21^{\text {st }}$ Annual International ACM SIGIR Conference on Research and Development in Information Retrieval. Melbourne, Australia, 37-45.

Barzilay, Regina, Kathleen R. McKeown, and Michael Elhadad. 1999. "Information Fusion in the Context of Multi-Document Summarization." Proceedings of the $37^{\text {th }}$ Association for Computational Linguistics. Maryland.

Cieri, Christopher. 2000. "Multiple Annotations of Reusable Data Resources: Corpora for Topic Detection and Tracking." Actes 5ième Journées Internationales d'Analyse Statistique des Données Textuelles (JADT).

Edmundson, H. P. 1969. "New Methods in Automatic Extracting." Journal for the Association for Computing Machinery 16 (2): 264-285.

Endres-Niggemeyer, Brigitte. 1998. Summarizing Information. Berlin: Springer-Verlag.

Goldstein, Jade, Vibhu Mittal, Jaime Carbonell, and Jamie Callan. 2000, November. "Creating and Evaluating Multi-Document Sentence Extract Summaries." Proceedings of the 2000 ACM CIKM International Conference on Information and Knowledge Management. McLean, VA, USA, 165-172. 
Grishman, Ralph. 2005, September. "NLP: An Information Extraction Perspective." Edited by Galia Angelova, Kalina Bontcheva, Ruslan Mitkov, Nicolas Nicolov, and Nikolai Nikolov, Recent Advances in Natural Language Processing (RANLP 2005). Borovets, Bulgaria: INCOMA, 1-4.

Jones, D., T. Bench-Capon, and P. Visser. 1998. "Methodologies for Ontology Development." Proceedings of the IT\&KNOWS Conference, XV IFIP World Computer Congress. Budapest.

Lehnert, Wendy G. 1981. "Plot Units: A Narrative Summarization Strategy." In Strategies for Natural Language Processing, edited by W. G. Lehnert and M. H. Ringle, 223-244. Hillsdale, New Jersey: Erlbaum. Also in (Mani and Maybury 1999),

Lopez, M. Fernadez. 1999. "Overview of Methodologies for Building Ontologies." Proceedings of the Workshop on Ontologies and Problem-Solving Methods: Lessons Learned and Future Trends (IJCAI99). Stockholm.

Luhn, H.P. 1958. "The Automatic Creation of Literature Abstracts." IBM Journal of Research 83 Development 2 (2): 159-165.

Mani, Inderjeet. 2001. Automatic Summarization. Volume 3 of Natural Language Processing. Amsterdam/Philadelphia: John Benjamins Publishing Company.

—. 2004. "Narrative Summarization." Journal Traitement Automatique des Langues (TAL): Special issue on "Le résumé automatique de texte: solutions et perspectives" 45, no. 1 (Fall).

Mani, Inderjeet, and Eric Bloedorn. 1999. "Summarizing Similarities and Differences Among Related Documents." Information Retrieval 1 (1): 123.

Mani, Inderjeet, and Mark T. Maybury, eds. 1999. Advances in Automatic Text Summarization. The MIT Press.

Mann, William C., and Sandra A. Thompson. 1987. "Rhetorical Structure Theory: A Framework for the Analysis of Texts." Technical Report ISI/RS87-185, Information Sciences Institute, Marina del Rey, California.

- 1988. "Rhetorical Structure Theory: Towards a Functional Theory of Text Organization." Text 8 (3): 243-281.

Marcu, Daniel. 1997. "The Rhetorical Parsing of Natural Language Texts." Proceedings of the $35^{\text {th }}$ Annual Meeting of the Association for Computational Linguistics. New Brunswick, New Jersey: Association for Computational Linguistics, 96-103.

- 2000. The Theory and Practice of Discourse Parsing and Summarization. The MIT Press.

—. 2001. "Discourse-Based Summarization in DUC-2001." Workshop on Text Summarization (DUC 2001). New Orleans.

Papka, Ron. 1999. "On-line New Event Detection, Clustering and Tracking." Ph.D. diss., Department of Computer Science, University of Massachusetts.

Petasis, G., V. Karkaletsis, D. Farmakiotou, I. Androutsopoulos, and C.D. SpyropoulosX. 2003. "A Greek Morphological Lexicon and its Exploitation by 
Natural Language Processing Applications." Edited by Yannis Manolopoulos, Skevos Evripidou, and Antonis Kakas, Advances in Informatics; Postproceedings of the 8th Panhellenic Conference in Informatics, Volume 2563 of Lecture Notes in Computer Science (LNCS). 401-419.

Petasis, George, Vangelis Karkaletsis, George Paliouras, Ion Androutsopoulos, and Costas D. Spyropoulos. 2002, May. "Ellogon: A New Text Engineering Platform." Proceedings of the 3rd International Conference on Language Resources and Evaluation (LREC 2002). Las Palmas, Canary Islands, Spain, 72-78.

Pinker, Steven. 1997. How the Mind Works. New York, London: W.W. Norton \& Company.

Pinto, H. S., and J. P. Martins. 2004. "Ontologies: How Can They Be Built?" Knowledge and Information Systems 6 (4): 441-464.

Radev, Dragomir R. 1999. "Generating Natural Language Summaries from Multiple On-Line Sources: Language Reuse and Regeneration." Ph.D. diss., Columbia University.

. 2000, October. "A Common Theory of Information Fusion from Multiple Text Sources, Step One: Cross-Document Structure." Proceedings of the 1st ACL SIGDIAL Workshop on Discourse and Dialogue. Hong Kong.

Radev, Dragomir R., and Kathleen R. McKeown. 1998. "Generating natural language summaries from multiple on-line sources." Computational Linguistics 24 (3): 469-500 (September).

Reiter, Ehud, and Robert Dale. 1997. "Building Applied Natural Language Generation Systems." Natural Language Engineering 3 (1): 57-87.

- 2000. Building Natural Language Generation Systems. Studies in Natural Language Processing. Cambridge University Press.

Salton, Gerald, Amit Singhal, Mandar Mitra, and Chris Buckley. 1997. "Automatic Text Structuring and Summarization." Information Processing and Management 33 (2): 193-207.

Stamatiou, George. 2005. "Extraction and Normalization of Temporal Expressions in the Context of Summarizing Evolving Events." Master's thesis, University of the Aegean.

Taboada, Maite, and William C. Mann. 2006. "Rhetorical Structure Theory: Looking Back and Moving Ahead." Discourse Studies 8 (3): 423-459.

Witten, Ian H., and Eibe Frank. 2000. Data Mining: Practical Machine Learning Tools and Techniques with Java Implementations. San Francisco: Morgan Kaufmann.

Zhang, Zhu, Sasha Blair-Goldensohn, and Dragomir Radev. 2002, August. "Towards CST-Enhanced Summarization." Proceedings of AAAI-2002.

Zhang, Zhu, Jahna Otterbacher, and Dragomir Radev. 2003, November. "Learning cross-document structural relationships using boosting." Proccedings of the Twelfth International Conference on Information and Knowledge Management CIKM 2003. New Orleans, Louisiana, USA, 124-130.

Zhang, Zhu, and Dragomir Radev. 2004, March. "Learning Cross-document Structural Relationships using Both Labeled and Unlabeled Data." Proceedings of IJC-NLP 2004. Hainan Island, China. 